\title{
Yes, Libor Models can capture Interest Rate Derivatives Skew : A Simple Modelling Approach
}

\author{
Eymen Errais * \\ Stanford University \\ Fabio Mercurio \\ Banca IMI.
}

January 11, 2005

\begin{abstract}
We introduce a simple extension of a shifted geometric Brownian motion for modelling forward LIBOR rates under their canonical measures. The extension is based on a parameter uncertainty modelled through a random variable whose value is drawn at an infinitesimal time after zero. The shift in the proposed model captures the skew commonly seen in the cap market, whereas the uncertain volatility component allows us to obtain more symmetric implied volatility structures.

We show how this model can be calibrated to cap prices. We also propose an analytical approximated formula to price swaptions from the cap calibrated model. Finally, we build the bridge between caps and swaptions market by calibrating the correlation structure to swaption prices, and analysing some implications of the calibrated model parameters.
\end{abstract}

\section{Introduction}

In the last decade, caps, swaptions, and other interest rate derivatives have become increasingly popular. According to the International Swaps and Derivatives Association, the transaction outstanding in interest rate swaps and options (and cross-currency swaps) at the half of 2004 stood at $\$ 164.49$ trillion in notional principal, up by more than 13 times from $\$ 11.303$ trillion at year-end 1994 .

The surge of trading volume in caps and swaptions urged the need for a theoretical justification for the related market formulas, which were derived by using the Black (1976) model in a seemingly unsound manner. The first major contribution toward this goal was accomplished by Heath Jarrow and Morton (HJM) (1992). HJM were the first to take instantaneous forward rates as state variables and to specify a general continuous time stochastic process for its evolution across time. The assumption that instantaneous rates exist is not always convenient, since it requires a certain degree of smoothness with respect to the maturity of bond prices and their volatilities. An alternative construction of an arbitrage-free family of bond prices, making no reference to the instantaneous, continuously compounded rates, is in some circumstances more suitable. This was achieved by Brace, Gatarek and Musiela (1997), Miltersen, Sandmann and Sondermann (1997) and Jamshidian (1997) who independently introduced what is known as Libor Market Model (LMM) ${ }^{1}$.

The LMM, which could be interpreted as a subset of the HJM class of models, was the first model to introduce a consistent theoretical framework for the market caps and swaptions formulas. In the LMM, one directly models market rates, namely the forward LIBOR rates, as driftless geometric Brownian motions under the associated forward measures. A major advantage of the LMM is the possibility of calibrating (at-the-money) caps volatilities automatically and (at-the-money) swaptions volatilities through efficient

\footnotetext{
*We are extremely grateful to Gianvittorio Mauri from Banca IMI for his helpful assistance in running the numerical tests. We are also grateful to John Weyant from Stanford University for his helpful comments. Useful discussions with Dariusz Gatarek from Numerix and helpful assistance from Andrea Pallavicini from Banca IMI are gratefully acknowledged.

${ }^{1}$ A different approach, but similar in spirit, was introduced by Santa-Clara and Sornette (2001) and Goldstein (2000) who proposed "String-Shocks" type of models to describe the evolution of forward rates.
} 
analytical approximations. These are the main reasons of the success of the LMM and explain why this model was quickly adopted by the industry. ${ }^{2}$

However, the LMM presents the major drawback of having deterministic volatility coefficients, which are not consistent with the skew or smile effects commonly seen both in the caps and the swaptions markets. To overcome this drawback, a natural extension of the Black-Scholes (1973) setting led to the introduction of Local Volatility Models (LVM), where volatility is a function of the underlying asset price (forward rate) and time. These models were pioneered by Dupire $(1993,1994)$ and Derman and Kani (1994) who introduced this extension for equity and foreign-exchange options. Andersen and Andreasen (2000) introduced a special case of LVM, the Constant Elasticity of Variance (CEV), to develop an extension of the LMM for capturing the skew. ${ }^{3}$

In other lines of research, Jamshidian (1999) and Glasserman and Kou (2003) introduced an alternative extension of the LMM by adding jumps in the forward rate dynamics. Besides presenting some technical complications, these models are unsuited to generate asymmetric smiles and skews since the jump component of the forward rate dynamics typically needs to be of substantial magnitude.

More recently, Andersen and Brotherton-Ratcliffe (2001), Andersen and Andreasen (2002), Joshi and Rebonato (2003) and Piterbarg (2003) introduced different families of Stochastic Volatility Models (SVM). In these models, each forward LIBOR rate follows a process with a time-dependent local volatility function, with a stochastic volatility perturbation uniformly applied to all LIBOR rates. A vast empirical study dedicated to stochastic volatility and jump LMM is given in Jarrow et al. (2004).

The models LVMs, LMMs with jumps or SVMs, however, are not the only extensions of the lognormal LMM allowing for smile- or skew-shaped implied volatilities. In fact it turns out that it is possible to introduce stochasticity in the volatility in a very simple and intuitive manner, so as to accommodate the market implied volatility surfaces while preserving a great deal of analytical tractability. This extension of the LMM was independently proposed by Gatarek (2003) and Brigo, Mercurio and Rapisarda (2004). In their approach, the forward rate dynamics are given by displaced geometric Brownian motions where the model parameters are not known at the initial time, but are discrete random variables whose values are drawn at an infinitesimal time. We refer to this model as to a Simple Extended Libor Model (SELM).

The shifts in the SELM are used to capture the skew effect commonly seen in the caps market, whereas the uncertain volatility component allows us to obtain more symmetric implied volatility structures. Accommodating the market smiles and skews is essential for complex-derivatives traders, who use plain-vanilla options to build their set of hedging instruments.

In this paper, we extend Gatarek's one-factor SELM to the multi-factor case and analyze its analytical tractability by deriving caps and swaptions prices in closed form. We then illustrate how the model can accommodate market caps data and how the instantaneous correlation parameters can be used for a calibration to swaptions prices. In fact, incorporating as much information as possible coming from swaptions quotes can be extremely important in the pricing of a number of exotic interest rate derivatives. To this end, we introduce a general and tractable correlation structure for forward rates to build the bridge between caps and swaptions and complete the "circle" of the joint calibration.

We finally analyze some important model's implications: i) we infer the swaptions smile implied by our joint calibration, and ii) we plot the evolution of some forward volatilities implied by the model.

The remainder of this paper is organized as follows. Section 2 sets up the characteristics of the SELM. Section 3 describes in detail how to perform the calibration to the caps market. Section 4 addresses the swaption valuation issue. Section 5 explains how to build the bridge between the caps and swaptions markets. Section 6 discusses some numerical results.

\footnotetext{
${ }^{2}$ In fact, many financial institution already used a discretized version of the Heath Jarrow and Morton (1992) model.

${ }^{3}$ As explained in Delbaen and Shirakawa (2002), the standard CEV model presents the drawback that its real world dynamics hit zero with positive probability.
} 


\section{The Model}

Let $\mathcal{T}=\left\{T_{0}, \ldots, T_{M}\right\}$ be a set of times and $\left\{\tau_{0}, \ldots, \tau_{M}\right\}$ the corresponding year fractions, meaning that $\tau_{k}$ is the year fraction for the interval $\left(T_{k-1}, T_{k}\right)$. We set $T_{-1}:=0$.

We consider a family of spanning forward rates $F_{k}$ with expiry $T_{k-1}$ and maturity $T_{k}, k=1, \ldots, M$ :

$$
F_{k}(t):=F\left(t ; T_{k-1}, T_{k}\right)=\frac{P\left(t, T_{k-1}\right)-P\left(t, T_{k}\right)}{\tau_{k} P\left(t, T_{k}\right)}
$$

with $P(t, T)$ denoting the time- $t$ price of the zero-coupon bond with maturity $T$.

We denote by $Q^{k}$ the $T_{k}$-forward measure, i.e. the probability measure associated with the numeraire $P\left(\cdot, T_{k}\right)$, and by $E^{k}$ the related expectation. We then denote by $Q^{d}$ the spot LIBOR measure, namely the probability measure associated with the discretely rebalanced bank-account numeraire $B_{d}$ :

$$
B_{d}(t)=\frac{P\left(t, T_{m-1}\right)}{\prod_{j=0}^{m-1} P\left(T_{j-1}, T_{j}\right)}, \quad T_{m-2}<t \leq T_{m-1}
$$

and by $E^{d}$ the related expectation. The measure $Q^{d}$ is the natural generalization of the classical risk-neutral measure to the LMM case, where the rates one models are simply compounded over finite time intervals.

By definition, the forward rate $F_{k}$ is a martingale under $Q^{k}$. To model the forward rates dynamics, therefore, is enough to model their diffusion coefficients. For instance, in a shifted lognormal LMM, one assumes the following diffusion coefficient for $F_{k}$ :

$$
\sigma_{k}(t)\left[F_{k}(t)+\alpha_{k}\right]
$$

where $\alpha_{k}$ is a constant and $\sigma_{k}$ is a deterministic function of time.

Once the volatility of forward rates has been defined, one then selects a particular measure under which considering the joint evolution of all rates. A quite common and convenient choice is the spot LIBOR measure, which is independent of the payoff to price. Since the drift of each forward rate under a given numeraire is readily obtained through the usual change of numeraire technique, one can directly postulate the joint evolution of all $F_{k}, k=1, \ldots, M$, under $Q^{d}$.

Let us now assume that the forward rates $F_{k}, k=1, \ldots, M$, evolve under $Q^{d}$ as in the SELM. The SELM was first introduced by Gatarek (2003) under a one-factor formulation. ${ }^{4}$ In this paper, we straightforwardly extend Gatarek's approach to the multi-factor case. Precisely, we assume that:

$$
d F_{k}(t)=\sigma_{k}^{I}(t)\left(F_{k}(t)+\alpha_{k}^{I}\right) \sum_{j=\beta(t)}^{k} \frac{\tau_{j} \rho_{j, k} \sigma_{j}^{I}(t)\left(F_{j}(t)+\alpha_{j}^{I}\right)}{1+\tau_{j} F_{j}(t)} d t+\sigma_{k}^{I}(t)\left(F_{k}(t)+\alpha_{k}^{I}\right) d Z_{k}^{d}(t)
$$

where

- $\beta(t)=\min \left\{i: t<T_{i-1}\right\}$, namely $\beta(t)=m$ if $T_{m-2} \leq t<T_{m-1}$;

- $Z^{d}$ is an $M$-dimensional Brownian motion with $d Z_{i}^{d}(t) d Z_{j}^{d}(t)=\rho_{i, j} d t$;

- $I$ is a random variable that is independent of $Z^{d}$ and takes values in the set $\{1,2, \ldots, N\}$ with $Q^{d}(I=$ $i)=\lambda_{i}, \lambda_{i}>0$ and $\sum_{i=1}^{N} \lambda_{i}=1$. The value of the random index $I$ is drawn at time $t=0^{+}$, namely at an infinitesimal time after time zero;

- $\sigma_{k}^{i}$ are (given) deterministic functions;

- $\alpha_{k}^{i}$ are (given) real constants.

\footnotetext{
${ }^{4}$ Brigo, Mercurio and Rapisarda (2004) proposed a similar approach in a more general setting.
} 
The intuition behind this model is as follows. The SELM is a LIBOR model where the forward rates (instantaneous) volatilities and displacements parameters are unknown (at time 0 ) and one assumes different scenarios for them. The volatilities and the displacements will be known immediately after time 0 .

The initial lack of knowledge on the true parameters values, which are "magically" revealed an instant later we apply our pricing model, can be a rather disturbing feature, especially for somebody pretending meaningful dynamics also from an historical point of view. Our support to the SELM lies on two grounds. First, the SELM can be viewed as a simplified version of a more general (and realistic) regime-switching model, where the model parameters, following Markov chains, can switch continually from one state to another. For many practical applications, however, the general model is too cumbersome and assuming a unique instantaneous change is already enough for capturing lots of desirable market features. Second, we believe that a model should be judged also in terms of its implications and not only in terms of its assumptions. For instance, though the SELM implied volatilities flatten out immediately in the future, the forward volatilities retain a realistic shape and hedging with the model is not necessarily a gamble. In the following sections, we will provide some empirical evidence supporting our claims.

Besides its intuitive meaning, the SELM has several advantages. It enjoys a great deal of analytical tractability, is relatively easy to implement and is flexible enough to accommodate general implied volatility surfaces in the caps market. This will be explained in the following, where practical examples with market data will be also considered, along with the analysis of some possible implications of the model.

\section{Calibration to Caplets}

In this section we show how to calculate analytically prices of caplets in the SELM, and explain how to perform, accordingly, the calibration to market volatilities.

A $T_{k-1}$-caplet is an option on a future LIBOR rate, set at time $T_{k-1}$ and paid at time $T_{k}$. Its payoff at time $T_{k}$ can be written as

$$
\tau_{k}\left[F_{k}\left(T_{k-1}\right)-K\right]^{+},
$$

so that the caplet can also be viewed as an option on the related forward rate.

Proposition 1 Under the SELM framework, the price of a caplet as described above is given by

$$
C p l^{\operatorname{SELM}}\left(T_{k-1}, T_{k}, \tau_{k}, K\right)=\tau_{k} P\left(0, T_{k}\right) \sum_{i=1}^{N} \lambda_{i} B l^{\text {call }}\left(K+\alpha_{k}^{i}, F_{k}(0)+\alpha_{k}^{i}, V_{k}^{i}\left(T_{k-1}\right)\right)
$$

where $V_{k}^{i}\left(T_{k-1}\right)=\sqrt{\int_{0}^{T_{k-1}} \sigma_{k}^{i}(s)^{2} d s}$ and $\operatorname{Bl}^{\text {call }}(K, F, V)$ is the Black price of a call with a strike $K$, forward price $F$, volatility $V$, maturity 1 and risk free rate 0 :

$$
B l^{\text {call }}(K, F, V)=F \Phi\left(\frac{\ln (F / K)+V^{2} / 2}{V}\right)-K \Phi\left(\frac{\ln (F / K)-V^{2} / 2}{V}\right),
$$

where $\Phi$ denotes the standard normal distribution function. An analogous definition holds for $B l^{p u t}$.

Proof. From (1), we immediately get that the dynamics of $F_{k}$ under $Q^{k}$ are

$$
d F_{k}(t)=\sigma_{k}^{I}(t)\left(F_{k}(t)+\alpha_{k}^{I}\right) d Z_{k}(t),
$$

where $Z_{k}$ is a Brownian motion under $Q^{k}$, and $I$ has under $Q^{k}$ the same distribution as under $Q^{d}{ }^{5}$

Standard no-arbitrage pricing implies that the caplet price at time $t=0$ is:

$$
\mathrm{Cpl}^{\mathrm{SELM}}\left(T_{k-1}, T_{k}, \tau_{k}, K\right)=\tau_{k} P\left(0, T_{k}\right) E^{k}\left[\left(F_{k}\left(T_{k-1}\right)-K\right)^{+}\right],
$$

\footnotetext{
${ }^{5}$ This is due to the independence between $Z^{d}$ and $I$.
} 
which, by applying an iterated conditioning, reads as

$$
\begin{aligned}
\operatorname{Cpl}^{\text {SELM }}\left(T_{k-1}, T_{k}, \tau_{k}, K\right) & =\tau_{k} P\left(0, T_{k}\right) E^{k}\left[E^{k}\left[\left(F_{k}\left(T_{k-1}\right)-K\right)^{+} \mid I\right]\right] \\
& =\tau_{k} P\left(0, T_{k}\right) E^{k}\left[E^{k}\left[\left(F_{k}\left(T_{k-1}\right)+\alpha_{k}^{I}-\left(K+\alpha_{k}^{I}\right)\right)^{+} \mid I\right]\right] \\
& =\tau_{k} P\left(0, T_{k}\right) \sum_{i=1}^{N} \lambda_{i} E^{k}\left[\left(F_{k}\left(T_{k-1}\right)+\alpha_{k}^{I}-\left(K+\alpha_{k}^{I}\right)\right)^{+} \mid I=i\right]
\end{aligned}
$$

Since $F_{k}$ evolves according to (3), conditional on $I, F_{k}\left(T_{k-1}\right)+\alpha_{k}^{I}$ is a lognormal random variable. The last expectation, therefore, is nothing but the adjusted Black's caplet price that comes from a shifted geometric Brownian motion (GBM).

To describe the calibration problem one has to deal with, we remember that, in the market, caplets are priced through Black's formula:

$$
\mathrm{Cpl}^{\mathrm{Mkt}}\left(T_{k-1}, T_{k}, \tau_{k}, K\right)=\tau_{k} P\left(0, T_{k}\right) \mathrm{Bl}^{\mathrm{call}}\left(K, F_{k}(0), V^{\mathrm{impl}}\left(T_{k-1}, K\right) \sqrt{T_{k-1}}\right)
$$

where $V^{\text {impl }}\left(T_{k-1}, K\right)$ is the market implied volatility of a caplet maturing at $T_{k-1}$ with strike $K$.

Setting $\Phi_{k}:=\left(V_{k}^{1}\left(T_{k-1}\right), \ldots, V_{k}^{N}\left(T_{k-1}\right), \alpha_{k}^{1}, \ldots, \alpha_{k}^{N}\right)$, the calibration to market caplets data is achieved by determining $\Phi_{k}$ as follows:

$$
\Phi_{k}=\arg \min \left[\sum_{j} g\left(\mathrm{Cpl}^{\mathrm{Mkt}}\left(T_{k-1}, T_{k}, \tau, K_{j}\right), \mathrm{Cpl}^{\mathrm{SELM}}\left(T_{k-1}, T_{k}, \tau, K_{j}\right)\right)\right],
$$

where the sum is taken over the set of strikes available for the maturity $T_{k}$ ( $T_{k-1}$-caplets), and $g$ is some "distance" function. In our practical examples, we will set $g(x, y)=(x / y-1)^{2}$. The parameters $\Phi_{k}$ are found iteratively starting from $k=1$ up to $k=M$, for some a priori given values of the probabilities $\lambda_{i}$.

This optimization produces as output the integral of instantaneous volatilities and not their point value. Hence, we have to introduce some assumptions on the volatility functions and infer their value from the calibrated integrals $V_{k}^{i}$. For instance, if we assume the $\sigma_{k}^{i}$ 's to be constant, we come up with the following formula:

$$
\sigma_{k}^{i}=\frac{V_{k}^{i}\left(T_{k-1}\right)}{\sqrt{T_{k-1}}}
$$

Another possibility is to assume a functional form like $\sigma_{k}^{i}(t)^{2}:=\left[a\left(T_{k-1}-t\right)+d\right] \exp \left(-b\left(T_{k-1}-t\right)\right)+c$. In this case, to find the parameters $a, b, c$ and $d$, we need to solve

$$
V_{k}^{i}\left(T_{k-1}\right)=\int_{0}^{T_{k-1}}\left(\left[a\left(T_{k-1}-t\right)+d\right] \exp \left(-b\left(T_{k-1}-t\right)\right)+c\right) d t
$$

For simplicity, in this paper we adopted the first (non parametric) assumption. An example of calibration to real market data will be considered in Section 6.1.1.

\section{Pricing Swaptions}

The swaptions market is the second main interest rate market. Swaptions are options on interest rate swaps and are priced in the market by means of a Black-like formula, see for instance Brigo and Mercurio (2001).

Many interest rate derivatives depend explicitly on swap rates or implicitly on the correlation between different LIBOR rates. To consistently price them, it is advisable to incorporate, in the valuation process, as much information as possible coming from the quoted swaption prices.

Obtaining accurate and fast approximations to the prices of European swaptions is crucial for an efficient calibration. While swaption prices in a LIBOR model should be calculated through a Monte Carlo simulation, it is however possible to derive efficient analytical approximations for them. The same applies to the SELM, as we show in the following. 


\subsection{An Approximated Analytical Formula}

In this section, we propose an approximation of swaption prices based on the classical "freezing" technique, which was first employed in the plain LMM case: When expressing a forward swap rate as a weighted average of spanning forward rates, one "freezes" the weights at their time-0 value, so as to obtain a linear combination with constant parameters. This approximation is similar in spirit to the one proposed by Gatarek (2003) in the one-factor case.

Proposition 2 The price of a payer swaption with option and swap maturities being respectively $T_{\alpha}$ and $T_{\beta}$ is given by :

$$
P \operatorname{Swptn}(\alpha, \beta, K)^{S E L M}=\sum_{h=\alpha+1}^{\beta} \tau_{h} P\left(0, T_{h}\right) \sum_{i=1}^{N} \lambda_{i} B l^{\text {call }}\left[K+\eta_{\alpha, \beta}^{i}, S_{\alpha, \beta}(t)+\eta_{\alpha, \beta}^{i}, \Gamma_{\alpha, \beta}^{i}\right]
$$

where $\Gamma_{\alpha, \beta}^{i}=\sqrt{\sum_{k, h=\alpha+1}^{\beta} \rho_{k, h} \int_{0}^{T_{\alpha}} \gamma_{k}^{i}(s) \gamma_{h}^{i}(s) d s}, \gamma_{k}^{I}(t)=\frac{\tau_{k} P\left(0, T_{k}\right) \sigma_{k}^{I}(t)\left(F_{k}(0)+\alpha_{k}^{I}\right)}{\sum_{h=\alpha+1}^{\beta} \tau_{h} P\left(0, T_{h}\right)\left(F_{h}(0)+\alpha_{h}^{I}\right)}, \eta_{\alpha, \beta}^{I}=\frac{\sum_{h=\alpha+1}^{\beta} \tau_{h} P\left(0, T_{h}\right) \alpha_{h}^{I}}{\sum_{h=\alpha+1}^{\beta} \tau_{h} P\left(0, T_{h}\right)}$, $\lambda_{i}$ is the probability of the scenario $i$ and $B l^{\text {call }}$ is defined by formula (2).

Analogously, the price of a receiver swaption is given by

$$
R \operatorname{Swptn}(\alpha, \beta, K)^{S E L M}=\sum_{h=\alpha+1}^{\beta} \tau_{h} P\left(0, T_{h}\right) \sum_{i=1}^{N} \lambda_{i} B l^{p u t}\left[K+\eta_{\alpha, \beta}^{i}, S_{\alpha, \beta}(t)+\eta_{\alpha, \beta}^{i}, \Gamma_{\alpha, \beta}^{i}\right]
$$

Proof. The forward swap rate $S_{\alpha, \beta}(t)$ at time $t$ for the set of times $T_{\alpha}, \ldots, T_{\beta}$ is defined by

$$
S_{\alpha, \beta}(t)=\frac{P\left(t, T_{\alpha}\right)-P\left(t, T_{\beta}\right)}{\sum_{k=\alpha+1}^{\beta} \tau_{k} P\left(t, T_{k}\right)}
$$

which can be written as a linear combination of consecutive forward rates as:

$$
S_{\alpha, \beta}(t)=\frac{\sum_{k=\alpha+1}^{\beta} \tau_{k} P\left(t, T_{k}\right) F_{k}(t)}{\sum_{k=\alpha+1}^{\beta} \tau_{k} P\left(t, T_{k}\right)}=\sum_{k=\alpha+1}^{\beta} \omega_{k}(t) F_{k}(t)
$$

where

$$
\omega_{k}(t)=\frac{\tau_{k} P\left(t, T_{k}\right)}{\sum_{h=\alpha+1}^{\beta} \tau_{h} P\left(t, T_{h}\right)}
$$

The forward swap rate $S_{\alpha, \beta}(t)$ is a martingale under the forward swap measure $Q^{\alpha, \beta}$, which is the measure associated with the numeraire $\sum_{k=\alpha+1}^{\beta} \tau_{k} P\left(t, T_{k}\right)$. Applying Ito's lemma, and noting that $S_{\alpha, \beta}(t)$ is a smooth function of the forward rates $F_{\alpha+1}, \ldots, F_{\beta}$, we obtain the following dynamics for $S_{\alpha, \beta}$ under the measure $Q^{\alpha, \beta}$ :

$$
d S_{\alpha, \beta}(t)=\sum_{k=\alpha+1}^{\beta} \frac{\partial S_{\alpha, \beta}(t)}{\partial F_{k}(t)} \sigma_{k}^{I}(t)\left(F_{k}(t)+\alpha_{k}^{I}\right) d Z_{k}^{\alpha, \beta}(t)=: \sum_{k=\alpha+1}^{\beta} \gamma_{k}(t) d Z_{k}^{\alpha, \beta}(t)
$$

The terms $\gamma_{k}$ can be approximated by neglecting the dependence of $\omega_{k}(t)$ on $F_{k}(t)$. We thus obtain:

$$
\begin{aligned}
\gamma_{k}(t) & \approx \frac{\tau_{k} P\left(t, T_{k}\right) \sigma_{k}^{I}(t)\left(F_{k}(t)+\alpha_{k}^{I}\right)}{\sum_{h=\alpha+1}^{\beta} \tau_{h} P\left(t, T_{h}\right)} \\
& =\frac{\tau_{k} P\left(t, T_{k}\right) \sigma_{k}^{I}(t)\left(F_{k}(t)+\alpha_{k}^{I}\right)}{\sum_{h=\alpha+1}^{\beta} \tau_{h} P\left(t, T_{h}\right)\left(F_{h}(t)+\alpha_{h}^{I}\right)} \frac{\sum_{h=\alpha+1}^{\beta} \tau_{h} P\left(t, T_{h}\right)\left(F_{h}(t)+\alpha_{h}^{I}\right)}{\sum_{h=\alpha+1}^{\beta} \tau_{h} P\left(t, T_{h}\right)} \\
& =\frac{\tau_{k} P\left(t, T_{k}\right) \sigma_{k}^{I}(t)\left(F_{k}(t)+\alpha_{k}^{I}\right)}{\sum_{h=\alpha+1}^{\beta} \tau_{h} P\left(t, T_{h}\right)\left(F_{h}(t)+\alpha_{h}^{I}\right)}\left[S_{\alpha, \beta}(t)+\frac{\sum_{h=\alpha+1}^{\beta} \tau_{h} P\left(t, T_{h}\right) \alpha_{h}^{I}}{\sum_{h=\alpha+1}^{\beta} \tau_{h} P\left(t, T_{h}\right)}\right] .
\end{aligned}
$$


This last expression can be further approximated by freezing the forward rates and the discount factors at their time 0 values:

$$
\begin{aligned}
\gamma_{k}(t) & \approx \frac{\tau_{k} P\left(0, T_{k}\right) \sigma_{k}^{I}(t)\left(F_{k}(0)+\alpha_{k}^{I}\right)}{\sum_{h=\alpha+1}^{\beta} \tau_{h} P\left(0, T_{h}\right)\left(F_{h}(0)+\alpha_{h}^{I}\right)}\left[S_{\alpha, \beta}(t)+\frac{\sum_{h=\alpha+1}^{\beta} \tau_{h} P\left(0, T_{h}\right) \alpha_{h}^{I}}{\sum_{h=\alpha+1}^{\beta} \tau_{h} P\left(0, T_{h}\right)}\right] \\
& =: \gamma_{k}^{I}(t)\left[S_{\alpha, \beta}(t)+\eta_{\alpha, \beta}^{I}\right]
\end{aligned}
$$

Therefore, the dynamics of $S_{\alpha, \beta}(t)$ under $Q^{\alpha, \beta}$ approximately reads as :

$$
\begin{aligned}
d S_{\alpha, \beta}(t) & =\sum_{k=\alpha+1}^{\beta} \gamma_{k}^{I}(t)\left[S_{\alpha, \beta}(t)+\eta_{\alpha, \beta}^{I}\right] d Z_{k}^{\alpha, \beta}(t) \\
& =\left[S_{\alpha, \beta}(t)+\eta_{\alpha, \beta}^{I}\right] \sum_{k=\alpha+1}^{\beta} \gamma_{k}^{I}(t) d Z_{k}^{\alpha, \beta}(t) \\
& =\gamma_{\alpha, \beta}^{I}(t)\left[S_{\alpha, \beta}(t)+\eta_{\alpha, \beta}^{I}\right] d W_{I}^{\alpha, \beta}(t)
\end{aligned}
$$

where $\gamma_{\alpha, \beta}^{I}(t)=\sqrt{\sum_{k, h=\alpha+1}^{\beta} \gamma_{k}^{I}(t) \gamma_{h}^{I}(t) \rho_{k, h}}$ and $d W_{I}^{\alpha, \beta}(t)=\frac{\sum_{k=\alpha+1}^{\beta} \gamma_{k}^{I}(t) d Z_{k}^{\alpha, \beta}(t)}{\gamma_{\alpha, \beta}^{I}(t)}$.

It can be verified easily that, conditional on $I, W_{I}^{\alpha, \beta}$ is a Brownian motion, so that if we define $X^{I}(t)$ by:

$$
X_{\alpha, \beta}^{I}(t):=S_{\alpha, \beta}(t)+\eta_{\alpha, \beta}^{I}
$$

we see that, conditional on $I, X_{\alpha, \beta}^{I}$ is a GBM whose dynamics is given by

$$
d X_{\alpha, \beta}^{I}(t)=\gamma_{\alpha, \beta}^{I}(t) X_{\alpha, \beta}^{I}(t) d W_{I}^{\alpha, \beta}(t)
$$

Having identified a GBM, we can easily price swaptions at time $t=0$, as we did for the previous caplet case.

A payer (resp. receiver) swaption with maturity $T_{\alpha}$, payment times $T_{\alpha}, \ldots, T_{\beta}$ and strike $K$ is the option to enter at time $T_{\alpha}$ an interest rate swap where, on each payment time $T_{k}$, we pay (resp. receive) the fixed rate $K$ and receive (resp. pay) the LIBOR rate $F_{k}\left(T_{k-1}\right)$. The payer swaption price can be calculated by taking expectation under the swap measure $Q^{\alpha, \beta}$ :

$$
\begin{aligned}
\operatorname{PSwptn}(\alpha, \beta, K)^{\mathrm{SELM}} & =\sum_{h=\alpha+1}^{\beta} \tau_{h} P\left(0, T_{h}\right) E^{\alpha, \beta}\left[\left(S_{\alpha, \beta}\left(T_{\alpha}\right)-K\right)^{+}\right] \\
& =\sum_{h=\alpha+1}^{\beta} \tau_{h} P\left(0, T_{h}\right) E^{\alpha, \beta}\left[\left(X_{\alpha, \beta}^{I}\left(T_{\alpha}\right)-\left(K+\eta_{\alpha, \beta}^{I}\right)\right)^{+}\right] \\
& =\sum_{h=\alpha+1}^{\beta} \tau_{h} P\left(0, T_{h}\right) \sum_{i=1}^{N} \lambda_{i} E^{\alpha, \beta}\left[\left(X_{\alpha, \beta}^{I}\left(T_{\alpha}\right)-\left(K+\eta_{\alpha, \beta}^{I}\right)\right)^{+} \mid I=i\right],
\end{aligned}
$$

where $E^{\alpha, \beta}$ denotes expectation under $Q^{\alpha, \beta}$. We obtain:

$$
\operatorname{PSwptn}(\alpha, \beta, K)^{\mathrm{SELM}}=\sum_{h=\alpha+1}^{\beta} \tau_{h} P\left(0, T_{h}\right) \sum_{i=1}^{N} \lambda_{i} \mathrm{Bl}^{\text {call }}\left[K+\eta_{\alpha, \beta}^{i}, S_{\alpha, \beta}(t)+\eta_{\alpha, \beta}^{i}, \Gamma_{\alpha, \beta}^{i}\right]
$$

where $\Gamma_{\alpha, \beta}^{i}=\sqrt{\int_{0}^{T_{\alpha}}\left[\gamma_{\alpha, \beta}^{i}(s)\right]^{2} d s}=\sqrt{\sum_{k, h=\alpha+1}^{\beta} \rho_{k, h} \int_{0}^{T_{\alpha}} \gamma_{k}^{i}(s) \gamma_{h}^{i}(s) d s}$.

Analogously, the price of a receiver swaption is given by

$$
\operatorname{RSwptn}(\alpha, \beta, K)^{\mathrm{SELM}}=\sum_{h=\alpha+1}^{\beta} \tau_{h} P\left(0, T_{h}\right) \sum_{i=1}^{N} \lambda_{i} \mathrm{Bl}^{\mathrm{put}}\left[K+\eta_{\alpha, \beta}^{i}, S_{\alpha, \beta}(t)+\eta_{\alpha, \beta}^{i}, \Gamma_{\alpha, \beta}^{i}\right]
$$

Similarly to what we have in the caplet case, therefore, the SELM swaption price is nothing but a mixture of adjusted Black's swaption prices. 


\subsection{Monte Carlo Swaption Price}

To verify the accuracy of our swaption price approximation, we compare the analytical formula (4) with the price obtained by Monte Carlo simulation. We now illustrate how this simulation is performed.

We use as reference measure the spot LIBOR measure $Q^{d}$. The swaption price, at time zero, under such a measure is given by:

$$
\operatorname{PSwptn}(\alpha, \beta, K)^{\mathrm{MC}}=E^{d}\left[\frac{\left(S_{\alpha, \beta}\left(T_{\alpha}\right)-K\right)^{+} \sum_{h=\alpha+1}^{\beta} \tau_{h} P\left(T_{\alpha}, T_{h}\right)}{B_{d}\left(T_{\alpha}\right)}\right] .
$$

This expectation can be calculated numerically by simulating the values, at time $T_{\alpha}$, of the forward rates spanning the swap rate interval:

$$
F_{\alpha+1}\left(T_{\alpha}\right), F_{\alpha+2}\left(T_{\alpha}\right), \ldots, F_{\beta}\left(T_{\alpha}\right) .
$$

These forward rates $F_{k}$ evolve under $Q^{d}$ according to (??), namely

$$
d F_{k}(t)=\sigma_{k}^{I}(t)\left(F_{k}(t)+\alpha_{k}^{I}\right) \sum_{j=\beta(t)}^{k} \frac{\tau_{j} \rho_{j, k} \sigma_{j}^{I}(t)\left(F_{j}(t)+\alpha_{j}^{I}\right)}{1+\tau_{j} F_{j}(t)} d t+\sigma_{k}^{I}(t)\left(F_{k}(t)+\alpha_{k}^{I}\right) d Z_{k}^{d}(t)
$$

where $Z^{d}$ is a $(\beta-\alpha)$-dimensional Brownian motion with $d Z_{i}^{d}(t) d Z_{j}^{d}(t)=\rho_{i, j} d t$. We can thus generate $F_{\alpha+1}\left(T_{\alpha}\right), F_{\alpha+2}\left(T_{\alpha}\right), \ldots, F_{\beta}\left(T_{\alpha}\right)$ by means of consecutive Euler approximations as follows:

$$
\begin{aligned}
F_{k}(t+\Delta t)-F_{k}(t)= & \sigma_{k}^{I}(t)\left(F_{k}(t)+\alpha_{k}^{I}\right) \sum_{j=\beta(t)}^{k} \frac{\tau_{j} \rho_{j, k} \sigma_{j}^{I}(t)\left(F_{j}(t)+\alpha_{j}^{I}\right)}{1+\tau_{j} F_{j}(t)} \Delta t \\
& +\sigma_{k}^{I}(t)\left(F_{k}(t)+\alpha_{k}^{I}\right)\left(Z_{k}^{\alpha}(t+\Delta t)-Z_{k}^{\alpha}(t)\right) .
\end{aligned}
$$

However, there exists a more efficient way to generate our forward rates. Set $\bar{F}_{k}^{\alpha, I}(t):=F_{k}(t)+\alpha_{k}^{I}$. By Ito's lemma, we have:

$$
d \ln \bar{F}_{k}^{\alpha, I}(t)=\sigma_{k}^{I}(t) \sum_{j=\beta(t)}^{k} \frac{\tau_{j} \rho_{j, k} \sigma_{j}^{I}(t) \bar{F}_{j}^{\alpha, I}(t)}{1+\tau_{j}\left(\bar{F}_{j}^{\alpha, I}(t)-\alpha_{j}^{I}\right)} d t-\frac{\sigma_{k}^{I}(t)^{2}}{2} d t+\sigma_{k}^{I}(t) d Z_{k}^{\alpha}(t) .
$$

This process has the advantage of having a deterministic diffusion coefficient, so that the Euler scheme for it coincides with the more sophisticated Milstein scheme. The interested reader is referred to Klöden and Platen (1995) for further developments on this subject.

Our simulation is performed by first randomly drawing a value $i$ of $I$ and then generating paths of the processes $\bar{F}_{k}^{\alpha, i}$ as follows:

$$
\begin{aligned}
\ln \bar{F}_{k}^{\alpha, i}(t+\Delta t)-\ln \bar{F}_{k}^{\alpha, i}(t)= & \sigma_{k}^{i}(t) \sum_{j=\beta(t)}^{k} \frac{\tau_{j} \rho_{j, k} \sigma_{j}^{i}(t) \bar{F}_{k}^{\alpha, i}(t)}{1+\tau_{j}\left(\bar{F}_{j}^{\alpha, i}(t)-\alpha_{j}^{i}\right)} \Delta t \\
& -\frac{\sigma_{k}^{i}(t)^{2}}{2} \Delta t+\sigma_{k}^{i}(t)\left(Z_{k}^{\alpha}(t+\Delta t)-Z_{k}^{\alpha}(t)\right) .
\end{aligned}
$$

Once the forward rates are simulated for the value $i$ of the random index, we can evaluate the normalized swaption payoff

$$
\frac{\left(S_{\alpha, \beta}\left(T_{\alpha} ; i\right)-K\right)^{+} \sum_{h=\alpha+1}^{\beta} \tau_{h} P\left(T_{\alpha}, T_{h} ; i\right)}{B_{d}\left(T_{\alpha} ; i\right)}
$$

along each trajectory and finally average the resulting values over all simulated paths. This leads to the Monte Carlo price of the swaption:

$$
\operatorname{PSwptn}(\alpha, \beta, K)^{\mathrm{MC}}=\frac{1}{n} \sum_{j=1}^{n} \frac{\left(S_{\alpha, \beta}\left(T_{\alpha} ; i, w_{j}\right)-K\right)^{+} \sum_{h=\alpha+1}^{\beta} \tau_{h} P\left(T_{\alpha}, T_{h} ; i, w_{j}\right)}{B_{d}\left(T_{\alpha} ; i, w_{j}\right)}
$$


where $w_{j}$ denotes the $j$-th path and $n$ is the total number of simulations.

To test the accuracy of our approximation, we will compare the analytical price (4) with the $99 \%$ Monte Carlo confidence interval:

$\operatorname{PSwptn}(\alpha, \beta, K)^{\mathrm{SELM}} \in\left[\operatorname{PSwptn}(\alpha, \beta, K)^{\mathrm{MC}}-2.576 \frac{\sigma_{\mathrm{PSwptn}^{\mathrm{MC}}}}{\sqrt{n}}, \operatorname{PSwptn}(\alpha, \beta, K)^{\mathrm{MC}}+2.576 \frac{\sigma_{\mathrm{PSwptn} \mathrm{MC}}}{\sqrt{n}}\right]$,

where $\sigma_{\mathrm{PSwptn}}{ }^{\mathrm{MC}}$ is the standard deviation of the Monte Carlo price.

The accuracy test can also be performed on implied volatilities rather than on prices. To this end, we recall that the swaption's market price, at time 0 , is given by Black's formula :

$$
\operatorname{PSwptn}(\alpha, \beta, K)^{\mathrm{Mkt}}=\mathrm{Bl}^{\text {call }}\left(K, S_{\alpha, \beta}(0), \sigma_{\alpha, \beta} \sqrt{T_{\alpha}}\right) \sum_{h=\alpha+1}^{\beta} \tau_{h} P\left(0, T_{h}\right) .
$$

The SELM implied volatility is defined as the parameter $\sigma_{\alpha, \beta}$ to plug into this market formula to match the corresponding SELM swaption price. The SELM implied volatility $\sigma_{\alpha, \beta}^{\text {SELM }}$ is thus implicitly defined by

$$
\mathrm{Bl}^{\text {call }}\left(K, S_{\alpha, \beta}(0), \sigma_{\alpha, \beta}^{\mathrm{SELM}} \sqrt{T_{\alpha}}\right)=\sum_{i=1}^{N} \lambda_{i} \mathrm{Bl}^{\text {call }}\left[K+\eta_{\alpha, \beta}^{i}, S_{\alpha, \beta}(0)+\eta_{\alpha, \beta}^{i}, \Gamma_{\alpha, \beta}^{i}\right]
$$

A similar definition applies to the Monte Carlo implied volatility $\sigma_{\alpha, \beta}^{\mathrm{MC}}$, which is implicitly defined by:

$$
\mathrm{Bl}^{\text {call }}\left(K, S_{\alpha, \beta}(0), \sigma_{\alpha, \beta}^{\mathrm{MC}} \sqrt{T_{\alpha}}\right) \sum_{h=\alpha+1}^{\beta} \tau_{h} P\left(0, T_{h}\right)=\operatorname{PSwptn}(\alpha, \beta, K)^{\mathrm{MC}}
$$

Numerical results and illustrations of these tests are presented in Section 6.2.

\section{Building the Bridge: Calibration to Swaptions}

As stated above, the first goal of the SELM is to capture the skew that is commonly seen in caps markets. After reaching this objective, we can move further and try to calibrate the model to at-the-money (ATM) swaption prices using the extra degrees of freedom left, namely the instantaneous correlations between the forward rates in our family. More precisely, after finding $\sigma_{k}^{I}$, s and $\alpha_{k}^{I}$ 's through a calibration to caplet prices, as explained in Section 3, we propose a methodology to find the correlation matrix best fitting the selected swaptions prices. Clearly, a joint calibration to both interest rate markets would require an overall optimization procedure, where the parameters $\sigma_{k}^{I}$ 's and $\alpha_{k}^{I}$ 's contribute to accommodate the swaptions implied volatilities. Here, instead, we resort to this two-stage procedure for simplicity, also because some model advantages are already evident in our simplified approach.

When calibrating correlations, our goal is not just to reproduce swaptions prices, but we would also like to provide a meaningful correlation structure that corresponds to market information. In fact, what matters in swaption pricing is the so-called terminal correlation and not the instantaneous one. Terminal correlation is a quantity summarizing the degree of "dependence" between forward rates at a future time, by contrast with instantaneous correlation which captures the "dependence" between (instantaneous) changes of different forward rates. The terminal correlation depends on the instantaneous correlations but also on the particular functions of time $\sigma_{k}^{I}(t)$ 's and $\alpha_{k}^{I}$ 's as well. We refer the reader to Rebonato (2004) for further developments on the subject.

A typical problem faced when we perform a joint calibration to caps and swaptions is the difference between fixed payment leg tenors. Indeed, our cap forward rates are semi-annual, whereas forward swap rates are annual. Therefore, we need to find a correspondence between 6 month $\sigma_{k, 5}^{I}$ cap parameters and their one year swaptions counterpart $\sigma_{k, 1}^{I}$. To this end, consider three caplets maturities $S, T$ and $N$, where $0<S<T<N$ 
and all 6 month spaced. Recall that the 6 month forward rates from $S$ to $T$ and from $T$ to $N$, and the annual forward rate from $S$ to $N$ can be computed respectively as follows: ${ }^{6}$

$$
F_{S, T}(t)=\frac{1}{.5}\left[\frac{P(t, S)}{P(t, T)}-1\right], F_{T, N}(t)=\frac{1}{.5}\left[\frac{P(t, T)}{P(t, N)}-1\right] \text { and } F_{S, N}(t)=\left[\frac{P(t, S)}{P(t, N)}-1\right]
$$

Therefore we have the following relation, see also Brigo and Mercurio (2001),

$$
F_{S, N}(t)=\frac{F_{S, T}(t)+F_{T, N}(t)}{2}+\frac{F_{S, T}(t) F_{T, N}(t)}{4}
$$

Applying Ito's lemma to both sides

$$
\begin{aligned}
d F_{S, N}(t)= & (\ldots) d t+\sigma_{S, .5}^{I}(t)\left(\frac{F_{S, T}(t)}{2}+\frac{F_{S, T}(t) F_{T, N}(t)}{4}\right) d Z_{T}(t) \\
& +\sigma_{T, .5}^{I}(t)\left(\frac{F_{T, N}(t)}{2}+\frac{F_{S, T}(t) F_{T, N}(t)}{4}\right) d Z_{N}(t),
\end{aligned}
$$

where $Z_{T}$ and $Z_{N}$ are two standard Brownian motions with instantaneous correlation $\rho_{T, N}$.

We assume, for the moment, that the shift parameters $\alpha$ are all zero. Equating the (instantaneous) quadratic variation of both sides in the last equation, and dividing $F_{S, N}^{2}(t)$, we obtain:

$$
\sigma_{S, 1}^{I}(t)^{2}=u_{1}(t)^{2} \sigma_{S, .5}^{I}(t)^{2}+u_{2}(t)^{2} \sigma_{T, .5}^{I}(t)^{2}+2 \rho_{T, N} u_{1}(t) u_{2}(t) \sigma_{S, .5}^{I}(t) \sigma_{T, .5}^{I}(t)
$$

where $u_{1}(t)=\frac{1}{F_{S, N}(t)}\left[\frac{F_{S, T}(t)}{2}+\frac{F_{S, T}(t) F_{T, N}(t)}{4}\right]$ and $u_{2}(t)=\frac{1}{F_{S, N}(t)}\left[\frac{F_{T, N}(t)}{2}+\frac{F_{S, T}(t) F_{T, N}(t)}{4}\right]$.

If we "freeze" all $F$ 's and hence all $u$ 's at their initial time zero value, we come up with the following approximation for the $\sigma$ parameters of the one-year long forward rate:

$$
\sigma_{S, 1}^{I}(t)^{2} \approx u_{1}(0)^{2} \sigma_{S, .5}^{I}(t)^{2}+u_{2}(0)^{2} \sigma_{T, 5}^{I}(t)^{2}+2 \rho_{T, N} u_{1}(0) u_{2}(0) \sigma_{S, .5}^{I}(t) \sigma_{T, .5}^{I}(t)
$$

To determine the $\alpha$ parameters of the annual forward rate $F_{S, N}$, we simply set $\alpha_{S, 1}^{I}=\alpha_{S, .5}^{I}$ where $\alpha_{S, .5}^{I}$ is the $\alpha$ parameter for the semi-annual forward rate $F_{S, T}$. This is also motivated by a preliminary work showing that, on each scenario, the values of $\alpha_{k}^{I}$ are not too different (same order of magnitude).

After providing a rule for converting semi-annual parameters into annual ones, we now need to parameterize our instantaneous correlation matrix to proceed to the calibration to swaptions prices. Modelling the instantaneous correlation should be tackled with caution. First of all, obviously, one must maintain the properties of a correlation matrix $\rho=\left(\rho_{i, j}\right)_{i, j}$, namely :

1. Symmetry: $\rho_{i, j}=\rho_{j, i}$, for all $i$ and $j$.

2. Positive semi-definiteness: $x^{\prime} \rho x \geq 0$, for any $x \in \mathbb{R}^{M}$, where $x^{\prime}$ denotes the transpose of $x$.

3. Unitary diagonal elements: $\rho_{i, i}=1$ for every $i$.

Moreover, when it comes to forward rates, there are additional characteristics, based on empirical observation, we would like their correlations to possess. Among these properties, the correlation matrix should display some time decay, i.e. the correlation between forward rates should decrease as the time between maturities increases. Inspired by these features we propose the two-parameter correlation structure.

Proposition 3 For a approaching 0 or a approaching $\infty, \rho_{i, j}$ for any $i$ and $j \geq 0$ represents a correlation structure

$$
\rho_{i, j}=\bar{\rho}+(1-\bar{\rho}) \sin \left(\frac{\pi}{2} \exp \left(-a \frac{\left|T_{j}-T_{i}\right|}{\bar{T}}\right)\right)
$$

where $\bar{\rho}$ is the long term correlation coefficient, a is the (positive) "speed" of decorrelation, and $\bar{T}$ is the maximum distance between any two maturities. The functional form (9) is decreasing in the difference $\left|T_{j}-T_{i}\right|$ and can lead to sigmoid shapes in the correlations.

\footnotetext{
${ }^{6}$ We assume, for simplicity, that the year fractions for semi-annual and annual rates are 0.5 and 1 , respectively.
} 
Proof. Properties 1. and 3. are trivially verified. Property 2. needs to be treated more carefully. Indeed, we need to impose some conditions to preserve the positive semi-definiteness of the correlation matrix. For $\bar{\rho}>0$ and $a$ approaching zero, we can consider the following first order Taylor expansion:

$$
\begin{aligned}
\rho_{i, j} & \simeq \bar{\rho}+(1-\bar{\rho}) \sin \left(\frac{\pi}{2}\left(1-a \frac{\left|T_{j}-T_{i}\right|}{\bar{T}}\right)\right) \\
& \simeq \bar{\rho}+(1-\bar{\rho}) \cos \left(\frac{\pi}{2} a \frac{\left|T_{j}-T_{i}\right|}{\bar{T}}\right)
\end{aligned}
$$

so that we approximately have

$$
\rho_{i, j} \simeq \bar{\rho}+(1-\bar{\rho}) \cos \left(\theta_{j}-\theta_{i}\right)
$$

where $\theta_{l}=\frac{\pi}{2} a \frac{T_{l}}{\bar{T}}$, for $l \in\{i, j\}$.

Since $\cos \left(\theta_{j}-\theta_{i}\right)$ defines the structure of a two-rank correlation matrix, see Rebonato $(2004)$, then $\left(\rho_{i, j}\right)$ is a positive semi-definite matrix as well.

For $\bar{\rho}>0$ and $a$ approaching $\infty$, we instead obtain:

$$
\rho_{i, j} \simeq \bar{\rho}+(1-\bar{\rho})\left(\frac{\pi}{2} \exp \left(-a \frac{\left|T_{j}-T_{i}\right|}{\bar{T}}\right)-\frac{1}{6}\left(\frac{\pi}{2} \exp \left(-a \frac{\left|T_{j}-T_{i}\right|}{\bar{T}}\right)\right)^{3}\right) \simeq \bar{\rho}
$$

and hence $\left(\rho_{i, j}\right)$ is again positive semi-definite.

In our calibration to swaptions prices, we will also test the performance of Rebonato's parametrization of correlations:

$$
\rho_{i, j}=\bar{\rho}+(1-\bar{\rho}) \exp \left(-a\left|T_{j}-T_{i}\right|\right) .
$$

Notice that, for a large enough, the above Taylor expansion approximation shows that our correlation structure is similar to Rebonato's. However, the two formulations can be rather different for small $a$. This will result in a different fitting quality to the same set of swaptions prices, as we will se in the numerical examples provided at the end of the paper. ${ }^{7}$

The calibration to swaptions prices is finally performed as follows. We recall, from the previous section, the SELM and market formulas for a payer swaption price, and we define $\Psi$ to be the set of optimization parameters, i.e. we set $\Psi:=(\bar{\rho}, a)$. The calibration aims at finding $\Psi$ such that

$$
\Psi=\arg \min \left(\sum_{\alpha, \beta} g\left(\operatorname{PSwptn}(t, \alpha, \beta, K)^{\mathrm{Mkt}}, \operatorname{PSwptn}(t, \alpha, \beta, K)^{\mathrm{SELM}}\right)\right),
$$

where $\alpha$ and $\beta$ range over our selected ATM swaption maturities and tenors.

Some numerical results are presented in Section (6.1.2).

\section{Numerical Results}

In this section, we illustrate some examples of calibration of the SELM to caps and swaptions market data, along the lines we previously mentioned. We also show the results of our tests on the good performance of the approximating SELM formula for swaptions. We conclude by plotting a few swaptions volatility skews implied by the previous model calibration, and by showing the evolution of some forward caplet volatilities in the future.

\footnotetext{
${ }^{7} \mathrm{~A}$ more accurate calibration to swaptions prices would require a less parsimonious correlation parametrization. As already pointed out, this is however beyond the scope of this paper.
} 


\subsection{Calibration to Market Data}

We start by testing the SELM on the caps market by considering different numbers of scenarios. Our numerical experiments, based on market data, show that three scenarios are usually enough for a satisfactory calibration, even though the fitting quality worsen for high strikes and low maturities.

We then move to consider swaption volatilities. As previously mentioned, our purpose is not to perform a true joint calibration, but rather to infer reasonable forward rates correlations to test the SELM also in terms of the swaptions volatility skews it implies.

\subsubsection{Calibration to caplet quotes}

We calibrated our model to the caps volatility data quoted on 08/11/2004 in the Euro market, using caplets with a six-month tenor. The caplets strikes range from $2.5 \%$ to $7 \%$, and are 25 basis points spaced. The caplets maturities spectrum ranges from 1.5 to 14.5 years. The volatility data used is plotted in Figure 1 , while a sample of the same data is displayed in Table 1.

We used two, three and finally four different scenarios of SELM parameters $(N=2,3,4)$, and came to the conclusion that three scenarios are usually the best choice. In fact, a two-scenarios model does not seem to accommodate the caplets skew accurately. Moreover, the marginal improvement in the fitting quality implied by a four-scenario model tends to be negligible. ${ }^{8}$ Therefore, in the remainder of our numerical tests, we decided to stick to a three-scenario model with a-priori given probabilities $\lambda$. Preliminary work showed, in fact, that substantially equivalent results, in terms of fitting, can be achieved through different choices for the scenarios probabilities. In our experiments, we took $\lambda_{1}=.6, \lambda_{2}=.3$ and $\lambda_{3}=.1$, thus assuming a high probability scenario, an average one, and a low probability scenario.

The calibrated values of $\sigma_{k}^{I}$ and $\alpha_{k}^{I}$ in the three different scenarios, and for maturities up to 14.5 years, are displayed in Tables 2 and 3. The resulting SELM caplet volatilities are plotted in Figure ??. Figures 3 to 7, instead, show a graphical comparison between the market and SELM implied caplet volatilities for a few selected maturities, namely $2,3,5,10$ and 12 years.

Table 1: Sample of market caplet volatilities (in \%) by strike and maturity

\begin{tabular}{|c|c|c|c|c|c|c|c|}
\hline Strike(\%)-Maturity & $\mathbf{2}$ & $\mathbf{2 , 5}$ & $\mathbf{3}$ & $\mathbf{3 , 5}$ & $\mathbf{4}$ & $\mathbf{4 , 5}$ & $\mathbf{5}$ \\
\hline $\mathbf{2 , 5}$ & 26,32 & 25,68 & 24,77 & 23,99 & 23,53 & 23,18 & 23,04 \\
$\mathbf{2 , 7 5}$ & 25,04 & 24,43 & 23,6 & 22,85 & 22,44 & 22,13 & 22,03 \\
$\mathbf{3}$ & 23,97 & 23,33 & 22,55 & 21,82 & 21,46 & 21,17 & 21,11 \\
$\mathbf{3 , 2 5}$ & 23,12 & 22,4 & 21,65 & 20,9 & 20,59 & 20,3 & 20,28 \\
$\mathbf{3 , 5}$ & 22,62 & 21,64 & 20,89 & 20,11 & 19,82 & 19,52 & 19,53 \\
$\mathbf{3 , 7 5}$ & 22,66 & 21,09 & 20,31 & 19,43 & 19,16 & 18,84 & 18,86 \\
$\mathbf{4}$ & 22,81 & 21,04 & 20,04 & 18,91 & 18,62 & 18,26 & 18,28 \\
$\mathbf{4 , 2 5}$ & 23,075 & 21,17 & 20,11 & 18,795 & 18,42 & 17,87 & 17,86 \\
$\mathbf{4 , 5}$ & 23,34 & 21,3 & 20,18 & 18,68 & 18,22 & 17,48 & 17,44 \\
$\mathbf{4 , 7 5}$ & 23,7 & 21,54 & 20,36 & 18,795 & 18,3 & 17,5 & 17,4 \\
$\mathbf{5}$ & 24,06 & 21,78 & 20,54 & 18,91 & 18,38 & 17,52 & 17,36 \\
$\mathbf{5 , 2 5}$ & 24,49 & 22,07 & 20,78 & 19,09 & 18,52 & 17,61 & 17,42 \\
$\mathbf{5 , 5}$ & 24,94 & 22,4 & 21,04 & 19,3 & 18,68 & 17,74 & 17,51 \\
$\mathbf{5 , 7 5}$ & 25,43 & 22,76 & 21,34 & 19,54 & 18,88 & 17,89 & 17,63 \\
$\mathbf{6}$ & 25,94 & 23,14 & 21,65 & 19,8 & 19,1 & 18,06 & 17,77 \\
$\mathbf{6 , 2 5}$ & 26,48 & 23,55 & 21,99 & 20,08 & 19,33 & 18,26 & 17,93 \\
$\mathbf{6 , 5}$ & 27,04 & 23,97 & 22,35 & 20,38 & 19,59 & 18,47 & 18,11 \\
$\mathbf{6 , 7 5}$ & 27,62 & 24,42 & 22,73 & 20,7 & 19,86 & 18,7 & 18,3 \\
$\mathbf{7}$ & 28,23 & 24,89 & 23,13 & 21,03 & 20,15 & 18,95 & 18,51 \\
\hline
\end{tabular}

\footnotetext{
${ }^{8}$ This result was also shown in Brigo, Mercurio and Rapisarda (2000) who calibrated a lognormal mixture density to market foreign-exchange data.
} 
Table 2: The calibrated values of $\sigma_{k}^{I}$ for different maturities and scenarios

\begin{tabular}{|c|c|c|c|}
\hline Caplets maturities & Scenario 1 & Scenario 2 & Scenario 3 \\
\hline 1.5 & 0.16304434 & 0.06422773 & 0.06332962 \\
2 & 0.18921474 & 0.05634155 & 0.05316215 \\
2.5 & 0.16791712 & 0.05989887 & 0.05520289 \\
3 & 0.17362959 & 0.06458859 & 0.06557753 \\
3.5 & 0.15172758 & 0.03617556 & 0.03240995 \\
4 & 0.15829939 & 0.04999707 & 0.03960524 \\
4.5 & 0.14123047 & 0.04344321 & 0.03870800 \\
5 & 0.14422573 & 0.05366341 & 0.04914180 \\
5.5 & 0.12441415 & 0.05057469 & 0.04322650 \\
6 & 0.12480429 & 0.05173816 & 0.04849220 \\
6.5 & 0.10744960 & 0.03105830 & 0.02892788 \\
7 & 0.12148858 & 0.05141806 & 0.04410974 \\
7.5 & 0.10697440 & 0.04382249 & 0.04019843 \\
8 & 0.11604050 & 0.04996482 & 0.03892146 \\
8.5 & 0.10106829 & 0.04246569 & 0.04085141 \\
9 & 0.10789990 & 0.04728225 & 0.04185310 \\
9.5 & 0.09942587 & 0.04068057 & 0.03929327 \\
10 & 0.10961703 & 0.04656102 & 0.04349751 \\
10.5 & 0.09826417 & 0.04100323 & 0.04260043 \\
11 & 0.10773474 & 0.04301775 & 0.04531406 \\
11.5 & 0.09486490 & 0.03734212 & 0.04308718 \\
12 & 0.09552005 & 0.03850786 & 0.04121609 \\
12.5 & 0.09505042 & 0.03621755 & 0.03896017 \\
13 & 0.09450712 & 0.03610641 & 0.04549258 \\
13.5 & 0.08950882 & 0.03703596 & 0.03723106 \\
14 & 0.09231341 & 0.03448961 & 0.03534253 \\
14.5 & 0.08597086 & 0.03469210 & 0.03425724 \\
\hline
\end{tabular}

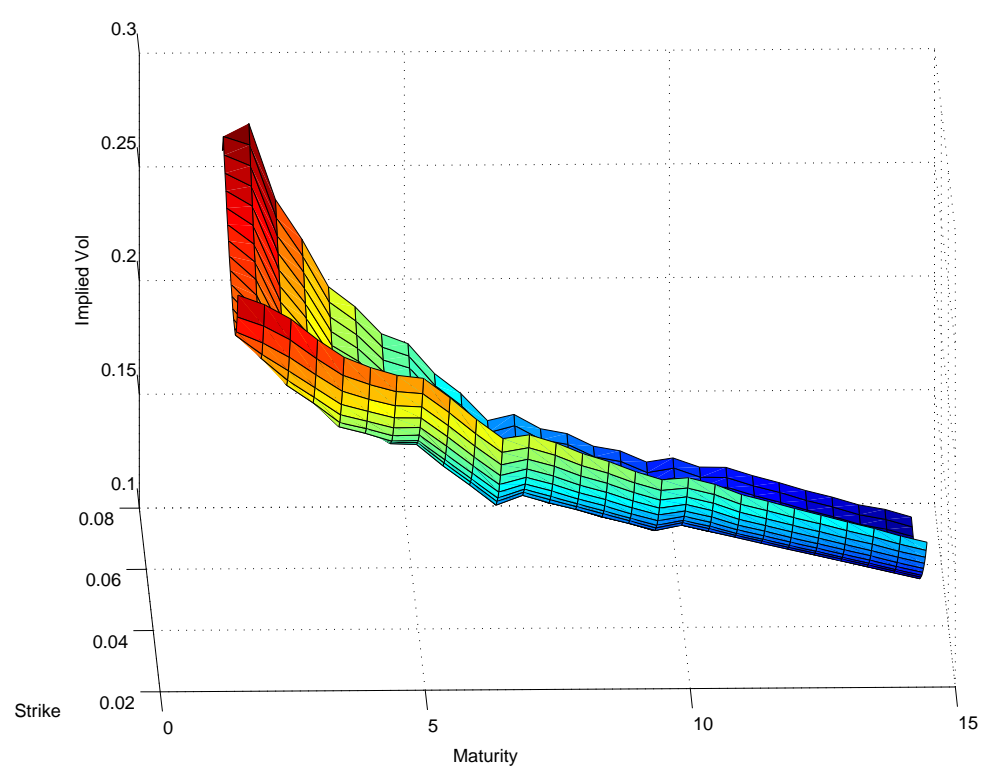

Figure 1: Market caplet volatilities as of 11 August 2004 by strike and maturity 
Table 3: The calibrated values of $\alpha_{k}^{I}$ for different maturities and scenarios

\begin{tabular}{|c|r|r|c|}
\hline Caplets maturities & Scenario 1 & Scenario 2 & Scenario3 \\
\hline 1.5 & 0.02801044 & 0.03841978 & 0.03326450 \\
2 & 0.02091647 & 0.03346086 & 0.03674928 \\
2.5 & 0.02596937 & 0.02426207 & 0.03236464 \\
3 & 0.02202359 & 0.01782716 & 0.02032976 \\
3.5 & 0.02776751 & 0.05817754 & 0.06401326 \\
4 & 0.02418964 & 0.02997589 & 0.04818130 \\
4.5 & 0.03074806 & 0.03732896 & 0.04390922 \\
5 & 0.02946808 & 0.02161852 & 0.02895950 \\
5.5 & 0.03719965 & 0.01976091 & 0.03170472 \\
6 & 0.03330999 & 0.01331947 & 0.01965747 \\
6.5 & 0.04104563 & 0.04570878 & 0.04912145 \\
7 & 0.03306023 & 0.01305767 & 0.01541584 \\
7.5 & 0.04193459 & 0.01881899 & 0.02348780 \\
8 & 0.03346430 & 0.01242679 & 0.01402056 \\
8.5 & 0.04336819 & 0.01611235 & 0.02000735 \\
9 & 0.03598035 & 0.00976178 & 0.01192281 \\
9.5 & 0.04088379 & 0.01448904 & 0.01933940 \\
10 & 0.03408434 & 0.00878642 & 0.01169428 \\
10.5 & 0.04182204 & 0.01214344 & 0.01838369 \\
11 & 0.03248840 & 0.00903381 & 0.01301825 \\
11.5 & 0.04134638 & 0.01417237 & 0.02011819 \\
12 & 0.03909778 & 0.01134578 & 0.01687170 \\
12.5 & 0.03775666 & 0.01239276 & 0.01803944 \\
13 & 0.03635414 & 0.00716251 & 0.01400981 \\
13.5 & 0.03920179 & 0.00779229 & 0.01037969 \\
14 & 0.03476033 & 0.00719563 & 0.02012456 \\
14.5 & 0.03879874 & 0.00730602 & 0.01047056 \\
\hline & & & \\
& & & \\
& &
\end{tabular}

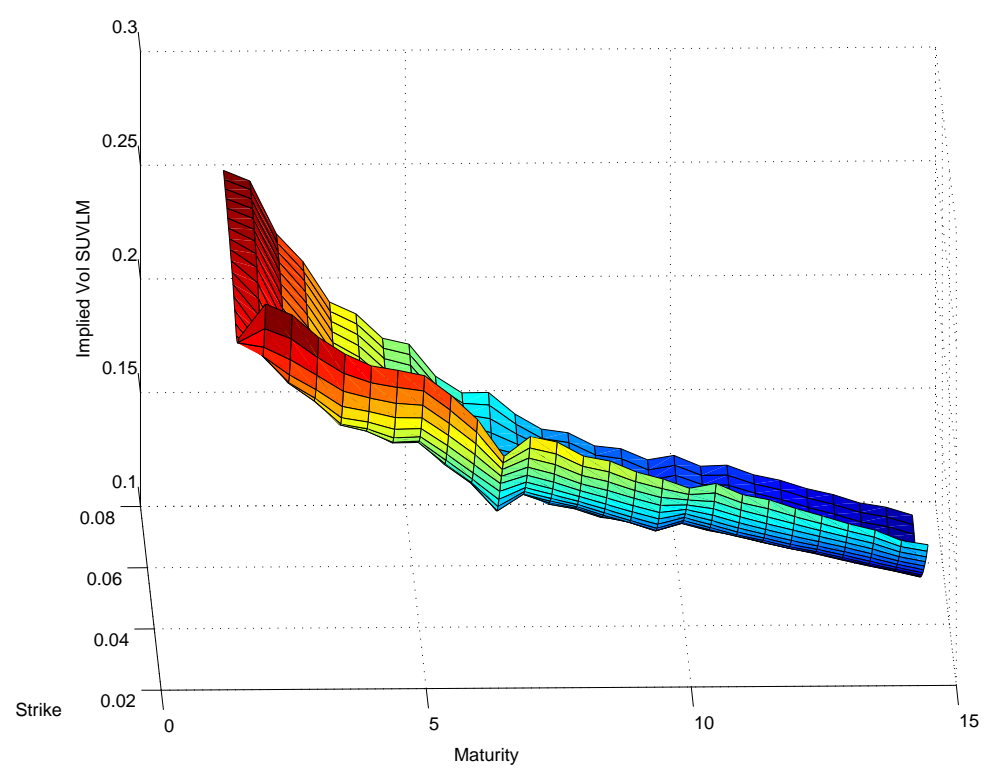

Figure 2: Calibration of the SELM model with three scenarios to the caplet skew for different maturities 


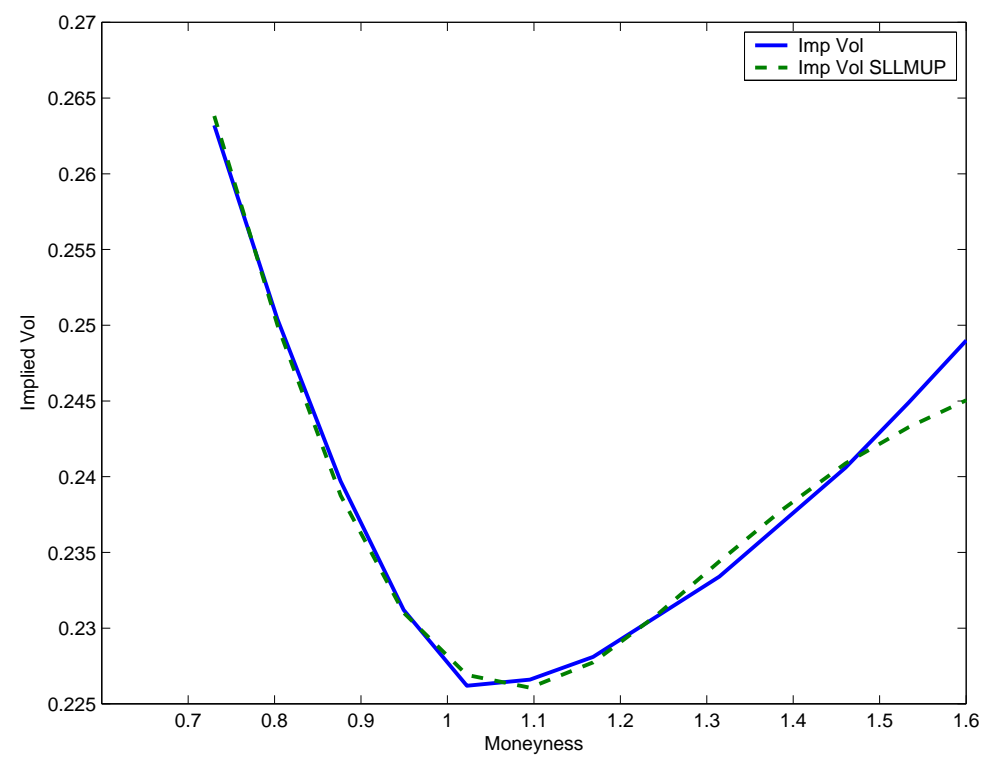

Figure 3: Calibration of the SELM to the two-year maturity caplet skew

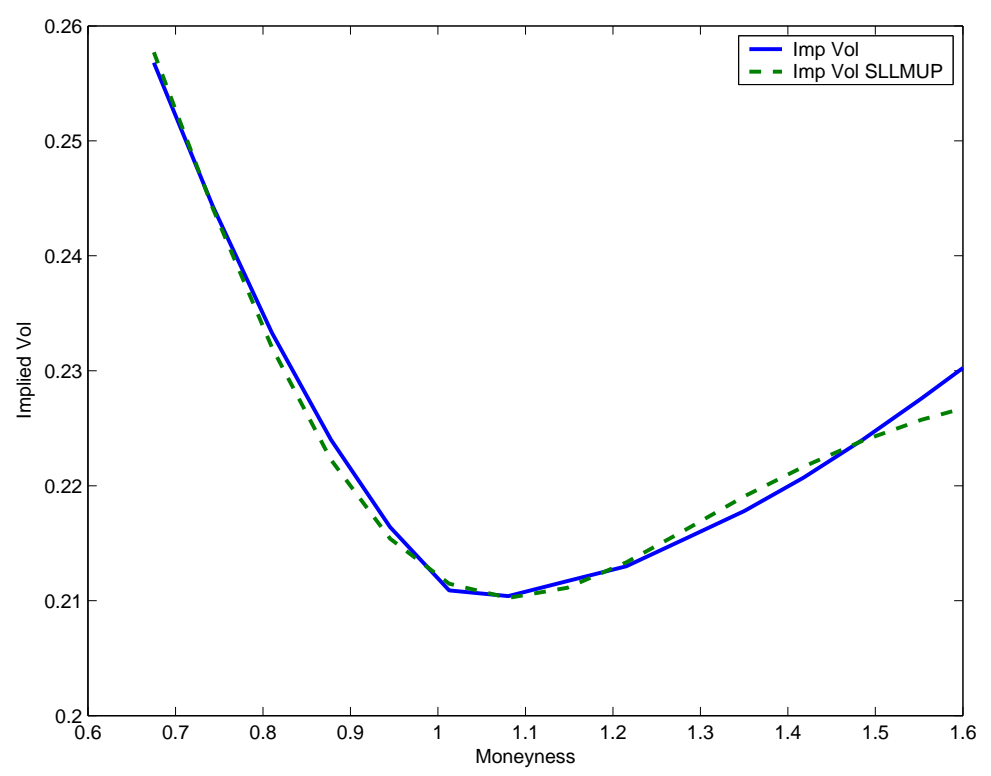

Figure 4: Calibration of the SELM to the three-year maturity caplet skew 


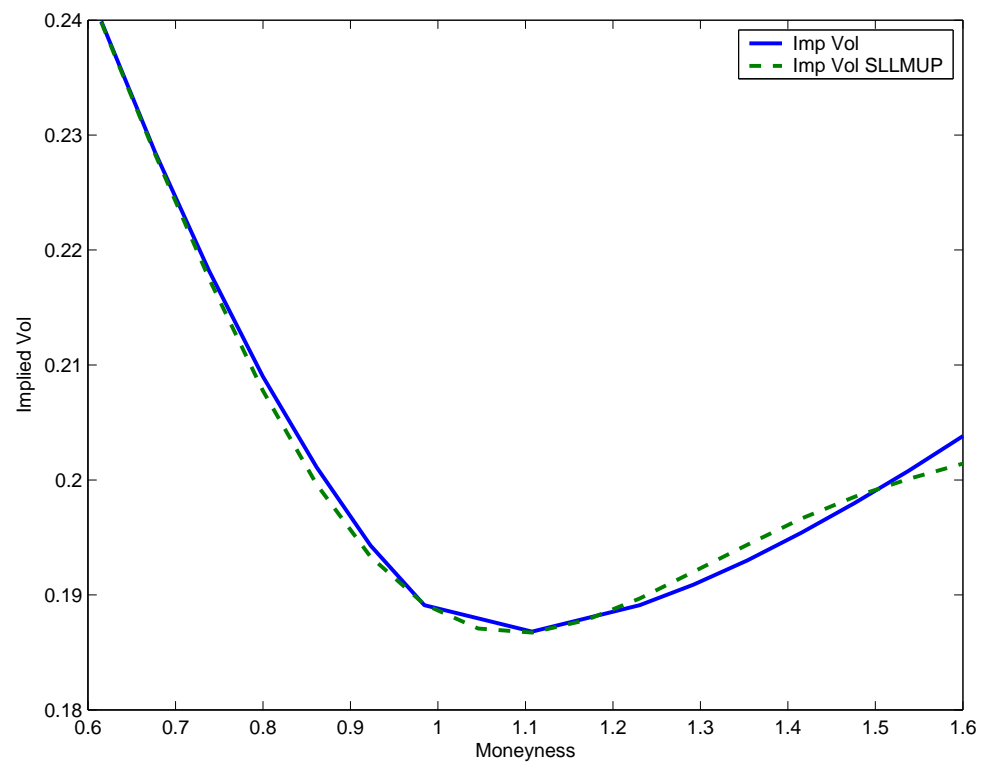

Figure 5: Calibration of the SELM to the five-year maturity caplet skew

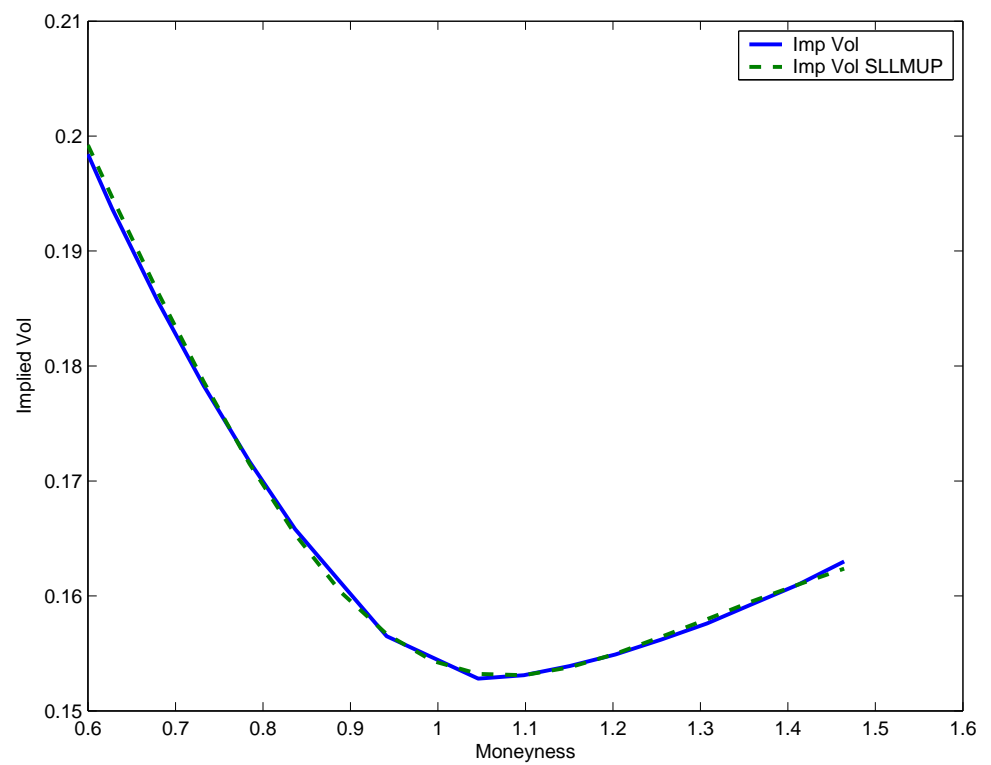

Figure 6: Calibration of the SELM to the ten-year maturity caplet skew 


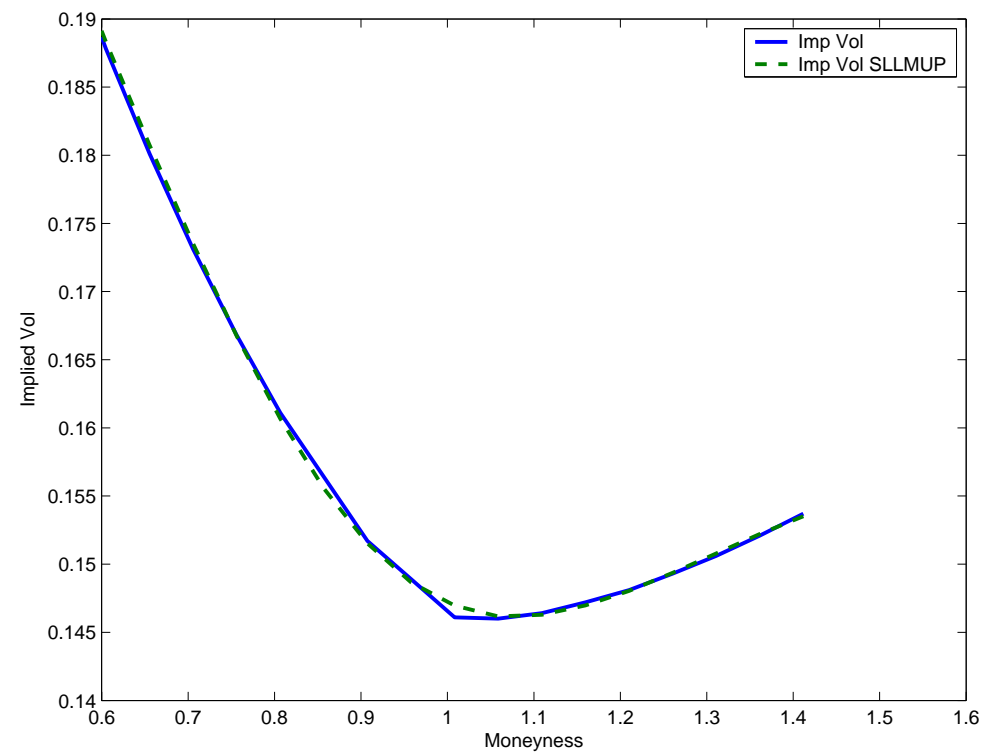

Figure 7: Calibration of the SELM to the twelve-year maturity caplet skew 


\subsubsection{Calibration to swaptions quotes}

We consider the ATM swaption volatilities quoted on 08/11/2004 in the Euro market. In the SELM swaption formula (4), the $\lambda$ parameters have been assigned a priori, whereas the $\alpha$ and $\sigma$ parameters for annual rates are obtained, as explained in Section 5, from the semi-annual ones coming from the previous calibration to caplets. The only free parameters are the correlations $\rho$, which are parameterized first through Rebonato's function (10), and then according to our form (9).

Table 4 displays the data used for the calibration. ${ }^{9}$ Tables 5 and 6 present the calibration results with Rebonato's formula, whereas Tables 7 and 8 present the results obtained with our correlation function.

Table 4: Market ATM swaption volatilities as of 11 August 2004

\begin{tabular}{|c|c|c|c|c|}
\hline Tenor-Swaption Maturity & $\mathbf{2 y}$ & $\mathbf{3 y}$ & $\mathbf{4 y}$ & $\mathbf{5 y}$ \\
\hline $\mathbf{2 Y}$ & 0.201 & 0.179 & 0.163 & 0.15 \\
$\mathbf{3 Y}$ & 0.187 & 0.168 & 0.153 & 0.142 \\
$\mathbf{4 Y}$ & 0.174 & 0.157 & 0.144 & 0.134 \\
$\mathbf{5 Y}$ & 0.163 & 0.147 & 0.135 & 0.127 \\
\hline
\end{tabular}

Table 5: SELM swaption volatility with Rebonato's correlation function

\begin{tabular}{|c|c|c|c|c|}
\hline Tenor-Swaption Maturity & $\mathbf{2 y}$ & $\mathbf{3 y}$ & $\mathbf{4 y}$ & $\mathbf{5 y}$ \\
\hline $\mathbf{2 Y}$ & 0.20268 & 0.18589 & 0.16783 & 0.15550 \\
$\mathbf{3 Y}$ & 0.18438 & 0.17016 & 0.15458 & 0.14416 \\
$\mathbf{4 Y}$ & 0.17016 & 0.15697 & 0.14401 & 0.13497 \\
$\mathbf{5 Y}$ & 0.15774 & 0.14632 & 0.13519 & 0.12671 \\
\hline
\end{tabular}

Table 6: Correlations under Rebonato's parametrization

\begin{tabular}{|c|c|}
\hline$\left|\boldsymbol{T}_{\boldsymbol{j}}-\boldsymbol{T}_{\boldsymbol{i}}\right|$ & $\boldsymbol{\rho}_{\boldsymbol{i}, \boldsymbol{j}}$ \\
\hline $\mathbf{0}$ & 1.00000 \\
$\mathbf{1}$ & 0.78098 \\
$\mathbf{2}$ & 0.61347 \\
$\mathbf{3}$ & 0.48535 \\
$\mathbf{4}$ & 0.38737 \\
$\mathbf{5}$ & 0.31244 \\
$\mathbf{6}$ & 0.25512 \\
$\mathbf{7}$ & 0.21129 \\
$\mathbf{8}$ & 0.17777 \\
$\mathbf{9}$ & 0.15213 \\
$\mathbf{1 0}$ & 0.13252 \\
$\mathbf{1 1}$ & 0.11752 \\
$\mathbf{1 2}$ & 0.10605 \\
\hline
\end{tabular}

Table 7: SELM swaption volatility with our correlation function

\begin{tabular}{|c|c|c|c|c|}
\hline Tenor-Swaption Maturity & $\mathbf{2 y}$ & $\mathbf{3 y}$ & $\mathbf{4 y}$ & $\mathbf{5 y}$ \\
\hline $\mathbf{2 Y}$ & 0.20040 & 0.18379 & 0.16591 & 0.15373 \\
$\mathbf{3 Y}$ & 0.18204 & 0.16799 & 0.15251 & 0.14224 \\
$\mathbf{4 Y}$ & 0.16895 & 0.15596 & 0.14298 & 0.13411 \\
$\mathbf{5 Y}$ & 0.15836 & 0.14699 & 0.13583 & 0.12726 \\
\hline
\end{tabular}

\footnotetext{
${ }^{9}$ The used data is just a small subsect of the matrix that is typically available in the market.
} 
Table 8: Correlations under our formulation

\begin{tabular}{|c|c|}
\hline$\left|\boldsymbol{T}_{\boldsymbol{j}}-\boldsymbol{T}_{\boldsymbol{i}}\right|$ & $\boldsymbol{\rho}_{\boldsymbol{i}, \boldsymbol{j}}$ \\
\hline $\mathbf{0}$ & 1.00000 \\
$\mathbf{1}$ & 0.74093 \\
$\mathbf{2}$ & 0.59764 \\
$\mathbf{3}$ & 0.55401 \\
$\mathbf{4}$ & 0.54125 \\
$\mathbf{5}$ & 0.53754 \\
$\mathbf{6}$ & 0.53646 \\
$\mathbf{7}$ & 0.53614 \\
$\mathbf{8}$ & 0.53605 \\
$\mathbf{9}$ & 0.53602 \\
$\mathbf{1 0}$ & 0.53601 \\
$\mathbf{1 1}$ & 0.53601 \\
$\mathbf{1 2}$ & 0.53601 \\
\hline
\end{tabular}




\subsection{Testing the Approximation for Swaptions Prices}

In this section we test the quality of the approximation (4) and the related implied volatility, by comparing their values with those coming from a Monte Carlo simulation.

In our test, we considered (payer) swaptions with a 5 year tenor. The SELM parameters used in the test are those coming from the previous calibrations to caps and swaptions, with instantaneous correlations parameterized by Rebonato's function.

Tables 9, 10, 11 and 12 present the results of our analysis. In each table, we report the approximation price, the $99 \%$ confidence interval of the Monte Carlo price, ${ }^{10}$ and the implied volatilities associated to the approximation and the Monte Carlo prices. These tables show that our approximation is quite accurate for every considered strike, both in terms of prices and implied volatilities.

Table 9: Approximation price, MC price-window and related volatilities for a $2 \times 5$ swaption

\begin{tabular}{|c|c|cc|c|c|}
\hline Strikes & Approx & Confidence & Interval & ImpVol Approx & ImpVol MC \\
\hline 1 & 13.82129 & 13.81992 & 13.82305 & 29.69013 & 30.93328 \\
1.5 & 11.7133 & 11.71163 & 11.7148 & 26.16912 & 26.09091 \\
2 & 9.616925 & 9.614254 & 9.617442 & 23.74041 & 23.53314 \\
2.5 & 7.558583 & 7.553763 & 7.556938 & 21.73473 & 21.53948 \\
3 & 5.585923 & 5.578241 & 5.581351 & 19.80649 & 19.64416 \\
3.5 & 3.76625 & 3.75582 & 3.758774 & 17.80746 & 17.6729 \\
4 & 2.237119 & 2.224806 & 2.227436 & 16.19233 & 16.07505 \\
4.5 & 1.205713 & 1.194609 & 1.196827 & 15.71271 & 15.61379 \\
5 & 0.652548 & 0.645185 & 0.647076 & 16.18942 & 16.11517 \\
5.5 & 0.361724 & 0.35747 & 0.359052 & 16.8489 & 16.79595 \\
6 & 0.19664 & 0.19451 & 0.195787 & 17.29857 & 17.26596 \\
6.5 & 0.103012 & 0.102198 & 0.103189 & 17.54427 & 17.53361 \\
7 & 0.052057 & 0.05184 & 0.052581 & 17.6619 & 17.67022 \\
\hline
\end{tabular}

Table 10: Approximation price, MC price-window and related volatilities for a $3 \times 5$ swaption

\begin{tabular}{|c|c|cc|c|c|}
\hline Strikes & Approx & Confidence & Interval & ImpVol Approx & ImpVol MC \\
\hline 1 & 14.41191 & 14.40977 & 14.41306 & 28.06964 & 26.61654 \\
1.5 & 12.39908 & 12.39643 & 12.39975 & 24.68383 & 24.24306 \\
2 & 10.402 & 10.39805 & 10.40138 & 22.36748 & 22.09171 \\
2.5 & 8.444132 & 8.438116 & 8.44144 & 20.50211 & 20.30066 \\
3 & 6.560787 & 6.552229 & 6.555507 & 18.79316 & 18.63539 \\
3.5 & 4.796158 & 4.785429 & 4.788599 & 17.09941 & 16.97469 \\
4 & 3.22747 & 3.215358 & 3.218309 & 15.58011 & 15.47835 \\
4.5 & 1.998144 & 1.986538 & 1.989155 & 14.68867 & 14.60644 \\
5 & 1.196614 & 1.187471 & 1.189798 & 14.59899 & 14.53471 \\
5.5 & 0.731471 & 0.725521 & 0.727613 & 14.9988 & 14.95317 \\
6 & 0.455184 & 0.451807 & 0.453648 & 15.46998 & 15.44153 \\
6.5 & 0.280946 & 0.279435 & 0.281013 & 15.81599 & 15.80503 \\
7 & 0.1699 & 0.169553 & 0.170871 & 16.02736 & 16.03384 \\
\hline
\end{tabular}

\footnotetext{
${ }^{10}$ We simulated ten million paths with a three-month time step. We used a predictor-corrector method for the drift of the forward rates and also applied major variance-reduction techniques.
} 
Table 11: Approximation price, MC price-window and related volatilities for a 4x5 swaption

\begin{tabular}{|c|c|cc|c|c|}
\hline Strikes & Approx & Confidence & Interval & ImpVol Approx & ImpVol MC \\
\hline 1 & 14.63908 & 14.63835 & 14.64162 & 26.82 & 27.69253 \\
1.5 & 12.72157 & 12.72022 & 12.72351 & 23.53826 & 23.61617 \\
2 & 10.82197 & 10.81942 & 10.82273 & 21.30599 & 21.22553 \\
2.5 & 8.960791 & 8.956455 & 8.959773 & 19.53616 & 19.43209 \\
3 & 7.16581 & 7.15942 & 7.162715 & 17.95743 & 17.85965 \\
3.5 & 5.468099 & 5.459839 & 5.463064 & 16.41676 & 16.33159 \\
4 & 3.913081 & 3.903392 & 3.906463 & 14.93485 & 14.86101 \\
4.5 & 2.603121 & 2.592899 & 2.595692 & 13.8419 & 13.77778 \\
5 & 1.658358 & 1.649555 & 1.652049 & 13.4545 & 13.40327 \\
5.5 & 1.067966 & 1.061611 & 1.063902 & 13.6628 & 13.62509 \\
6 & 0.707301 & 0.703289 & 0.705397 & 14.0972 & 14.07241 \\
6.5 & 0.471931 & 0.469642 & 0.471546 & 14.48584 & 14.47222 \\
7 & 0.311619 & 0.310611 & 0.312297 & 14.75378 & 14.75166 \\
\hline
\end{tabular}

Table 12: Approximation price, MC price-window and related volatilities for a 5x5 swaption

\begin{tabular}{|c|c|cc|c|c|}
\hline Strikes & Approx & Confidence & Interval & ImpVol Approx & ImpVol MC \\
\hline 1 & 14.59393 & 14.5914 & 14.59454 & 25.50006 & 24.68521 \\
1.5 & 12.76953 & 12.7665 & 12.76966 & 22.35811 & 22.04726 \\
2 & 10.96356 & 10.95958 & 10.96276 & 20.22934 & 20.04543 \\
2.5 & 9.193978 & 9.188817 & 9.192016 & 18.55779 & 18.43177 \\
3 & 7.483998 & 7.477476 & 7.480671 & 17.09149 & 16.99571 \\
3.5 & 5.85859 & 5.850897 & 5.854049 & 15.68794 & 15.61252 \\
4 & 4.350503 & 4.341947 & 4.344986 & 14.32488 & 14.26323 \\
4.5 & 3.03345 & 3.024703 & 3.027518 & 13.2165 & 13.16572 \\
5 & 2.017548 & 2.009981 & 2.012516 & 12.65815 & 12.61917 \\
5.5 & 1.337118 & 1.331628 & 1.333973 & 12.66687 & 12.63976 \\
6 & 0.908976 & 0.905317 & 0.907527 & 12.98943 & 12.97167 \\
6.5 & 0.628781 & 0.626678 & 0.628735 & 13.35872 & 13.35002 \\
7 & 0.434486 & 0.433625 & 0.435504 & 13.64837 & 13.64914 \\
\hline
\end{tabular}




\subsection{Further Model Implications}

Besides the possibility of accommodating caps and swaptions quotes, the SELM has two further appealing features. First, the SELM allows for a definition of a possible swaption market smile or skew. In practice, swaptions are quoted ATM and quotes for away from the money swaptions are rather rare. However, practitioners who trade out of the money (OTM) and in the money (ITM) swaptions agree on pricing them with a higher volatility than the ATM one. Therefore, in the absence of OTM and ITM quotes, our model can provide a guide for practitioners to produce their quotes.

Figure 8 shows the implied volatilities curves induced by our model for they the $2 \times 5,3 \times 5,4 \times 5,5 \times 5$ swaptions, with the same model parameters used in the previous section to test the SELM price formula. These plots should only be regarded as examples of possible implications of our model. In fact, more "realistic" curves could be obtained through a more accurate calibration to ATM swaptions prices.

Another important implication of our model is that it produces self-similar forward caplets volatilities. In fact, the requirement of a model implying forward volatility skews that are similar to the current ones is often neglected when modelling the evolution of forward rates, since fitting caplets and swaptions market data is a more obvious and compelling goal. Having realistic forward volatility skews is important as far as pricing exotic options is concerned since it allows traders to implement a better hedging strategy for their derivatives books.

A forward implied volatility is defined as the volatility parameter to plug into the Black-Scholes formula for forward starting option (FSO) to match the model price. ${ }^{11}$ A forward start caplet (FSC) with forward start date $T_{j}$ and maturity $T_{k}$ is an option on the LIBOR rate $F_{k}\left(T_{k-1}\right)$, with $T_{k-1}>T_{j}$, where the strike price is set as a proportion $\delta$ of the spot LIBOR rate at time $T_{j}$. In case of a call, the payoff at time $T_{k}$ is (we assume a unit nominal amount):

$$
\tau_{k}\left[F_{k}\left(T_{k-1}\right)-\delta F_{j+1}\left(T_{j}\right)\right]^{+} .
$$

This payoff, however, involves two different forward rates, and as such can not lead to a consistent definition of forward implied volatility. To this end, we replace (11) with the following:

$$
\tau_{k}\left[F_{k}\left(T_{k-1}\right)-\delta F_{k}\left(T_{j}\right)\right]^{+}
$$

The difference between the two formulations is that in the former the strike is defined by the spot LIBOR rate at time $T_{j}$ for maturity $T_{j+1}$, whereas in the latter formulation, it is defined by the time $T_{j}$ forward LIBOR rate between $T_{k-1}$ and $T_{k}$. Even though the payoff (12) is somehow more intuitive, it is not exchanged in the market essentially because forward rates are not directly quoted by the market but only stripped from zero-coupon bonds. The second payoff is also more convenient mathematically, since it is the difference between two values of the same "asset" computed at different times (in the former payoff we have to cope with two different LIBOR rates with two different dynamics). Having to define a forward volatility, and not to price a true market derivative, we thus stick to the payoff (12).

Following Rubinstein (1991), under a lognormal LMM, the price of a FSC at time 0 is given by

$$
\operatorname{FSCpl}\left(T_{j}, T_{k-1}, T_{k}, \tau_{k}, \delta\right)=\tau_{k} P\left(0, T_{k}\right) \mathrm{Bl}^{\text {call }}\left(\delta F_{k}(0), F_{k}(0), V^{\mathrm{impl}}\left(T_{j}, T_{k-1}\right) \sqrt{T_{k-1}-T_{j}}\right)
$$

where $V^{\mathrm{impl}}\left(T_{j}, T_{k-1}\right)$ is the time $T_{j}$-forward implied volatility for the expiry $T_{k-1}$.

\footnotetext{
${ }^{11}$ While in equity and foreign exchange markets FSOs are commonly traded, these derivatives are less present in the fixed income market.
} 
Similarly, the price of a FSC with the SELM is given by

$$
\begin{aligned}
& \mathrm{FSCpl}^{\mathrm{SELM}}\left(T_{j}, T_{k-1}, T_{k}, \tau_{k}, \delta\right)=\tau_{k} P\left(0, T_{k}\right) \sum_{i=1}^{N} \lambda_{i} E^{k}\left[\left(\left(F_{k}\left(T_{k-1}\right)+\alpha_{k}^{i}\right)-\left(\delta F_{k}\left(T_{j}\right)+\alpha_{k}^{i}\right)\right)^{+} \mid I=i\right] \\
& =\tau_{k} P\left(0, T_{k}\right) \sum_{i=1}^{N} \lambda_{i} E^{k}\left[E^{k}\left[\left(\left(F_{k}\left(T_{k-1}\right)+\alpha_{k}^{i}\right)-\left(\delta F_{k}\left(T_{j}\right)+\alpha_{k}^{i}\right)^{+}\right) \mid\left\{F_{k}\left(T_{j}\right), I=i\right\}\right] \mid I=i\right] \\
& =\tau_{k} P\left(0, T_{k}\right) \sum_{i=1}^{N} \lambda_{i} E^{k}\left[\mathrm{Bl}^{\text {call }}\left(\delta F_{k}\left(T_{j}\right)+\alpha_{k}^{i}, F_{k}\left(T_{j}\right)+\alpha_{k}^{i}, V_{T_{j}}^{i}\left(T_{k-1}\right)\right)\right] \\
& =\tau_{k} P\left(0, T_{k}\right) \sum_{i=1}^{N} \lambda_{i} \int_{-\infty}^{+\infty}\left[\mathrm{Bl}^{\text {call }}\left(\delta F_{k}\left(T_{j}\right)+\alpha_{k}^{i}, F_{k}\left(T_{j}\right)+\alpha_{k}^{i}, V_{T_{j}}^{i}\left(T_{k-1}\right)\right)\right] d \Phi\left(F_{k}\left(T_{j}\right)\right)
\end{aligned}
$$

where we set $V_{T_{j}}^{i}\left(T_{k-1}\right)=\sqrt{\int_{T_{j}}^{T_{k-1}} \sigma_{k}^{i}(s)^{2} d s}$ and $\Phi\left(F_{k}\left(T_{j}\right)\right)$ is the (lognormal) cumulative distribution function of $F_{k}\left(T_{j}\right)$ under $Q^{k}$.

The time $T_{j}$-forward implied volatility for the expiry $T_{k-1}$ is, by definition, the parameter $V^{\mathrm{impl}}\left(T_{j}, T_{k-1}\right)$ that satisfies:

$$
\operatorname{FSCpl}\left(T_{j}, T_{k-1}, T_{k}, \tau_{k}, \delta\right)=\mathrm{FSCpl}^{\mathrm{SELM}}\left(T_{j}, T_{k-1}, T_{k}, \tau_{k}, \delta\right) .
$$

To test the forward implied volatility generated by our model in a quick and simpler way, we assume the $\alpha_{k}^{I}$ 's to be equal to zero in each scenario. In this case the SELM formula simplifies to

$$
\operatorname{FSCpl}^{\mathrm{SELM}}\left(T_{j}, T_{k-1}, T_{k}, \tau, \delta\right)=\tau_{k} P\left(0, T_{k}\right) \sum_{i=1}^{N} \lambda_{i} \mathrm{Bl}^{\text {call }}\left(\delta F_{k}(0), F_{k}(0), V_{T_{j}}^{i}\left(T_{k-1}\right)\right)
$$

We priced FSCs starting in one year and having maturities 2, 3, 5 and 10 years. Figure 9 shows the results of our test.

From our analysis, the SELM seems to imply forward volatilities that are very similar to the current ones. Note also that a slight difference is due to the fact that we priced the FSCs by neglecting the $\alpha_{k}^{I}$ 's (the forward volatility graphs do not display a clear skew). 


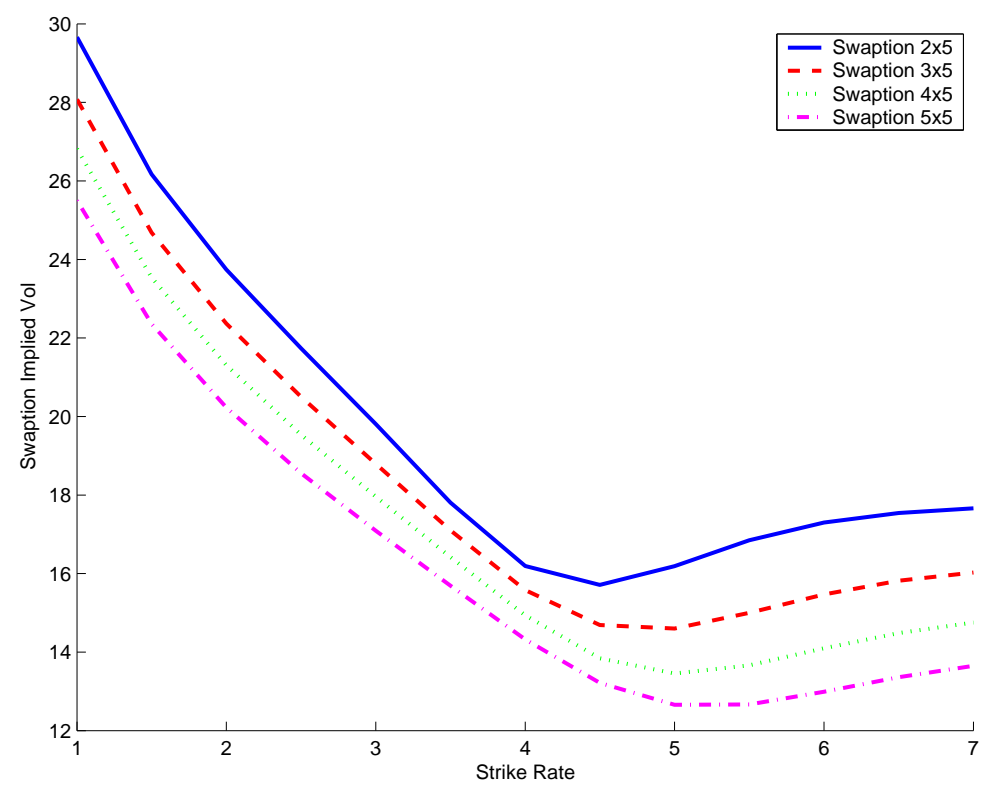

Figure 8: The implied smile for the $2 \times 5,3 \times 5,4 \times 5$ and $5 \times 5$ swaptions

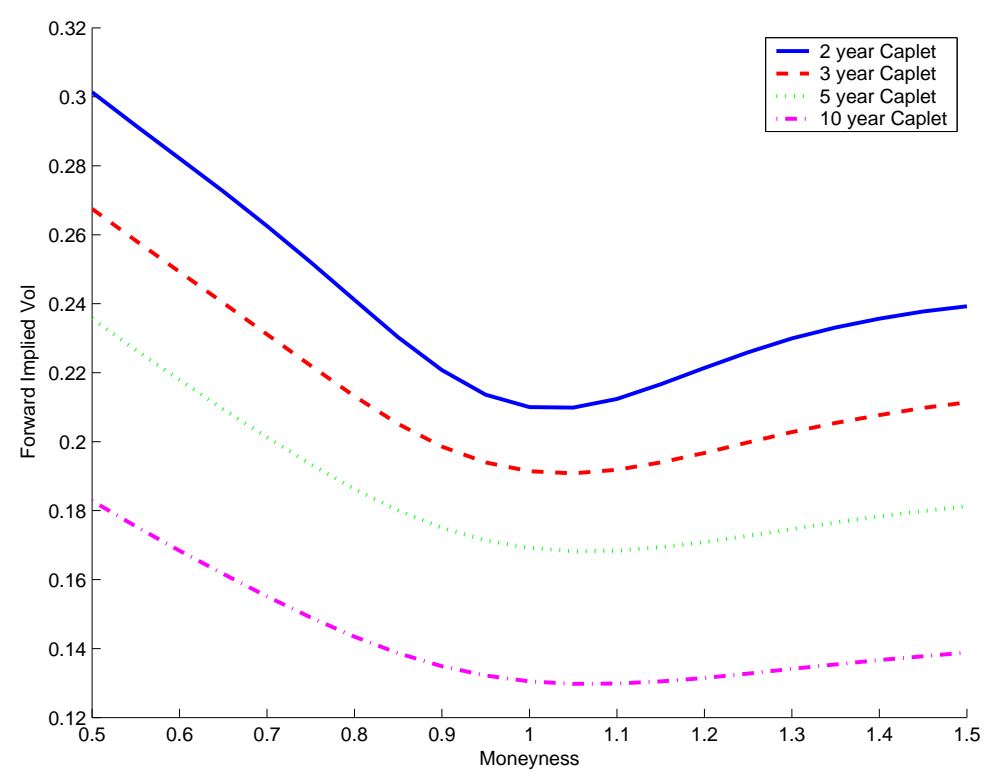

Figure 9: One year forward volatility smiles of two, three, five and ten year maturity caplets. 


\section{Conclusions}

Gatarek (2003) proposed a simple one-factor stochastic volatility LMM based on parameters uncertainty. In this paper, we have generalized Gatarek's approach to the multi factor case.

Besides being quite intuitive, the model is extremely easy to implement because of its tractability. In fact, caplets prices are simply equal to the weighted average of adjusted Black's caplet prices, and swaptions can be priced, in a similar fashion, through a closed-form approximation.

The model can accommodate a large variety of caplet volatility surfaces and can be simultaneously calibrated to ATM swaptions prices. This has been tested on real market data.

A seeming drawback of the model is that future implied volatilities flatten almost immediately, i.e. as soon as the random value of the model parameters is drawn a little after time zero. However, our empirical analysis shows that the forward implied volatilities induced by the model do not differ much from the current ones, providing a further support for its use in the pricing and hedging of interest rate derivatives.

Further empirical work can be carried out by trying different parametrizations for the time-dependent functions $\sigma_{k}^{I}$. These parametrizations can be different for different scenarios. Another interesting study concerns a true joint calibration to the caps and swaptions markets, with the analysis of related implications, especially in terms of the swaptions implied volatility smile or skew. 


\section{References}

[1] Andersen L., Andreasen J. (2000), Volatility skews and extensions of the Libor Market Model, Applied Mathematical Finance, 7:1-32, March 2000.

[2] Andersen, L., Andreasen J. (2002), Volatile volatilities, RISK, Volume 15, No. 12, p. 163-168.

[3] Andersen L., Brotherton-Ratcliffe R. (2001), Extended LIBOR market models with stochastic volatility, Working paper, Banc of America Securities.

[4] Andersen L., Piterbarg V. (2004), Moment Explosions in Stochastic Volatility Models, Working Paper, Bank of America.

[5] Black, F. (1976), The pricing of commodity contracts, Journal of Financial Economics, 3, 167-179

[6] Black F., Scholes M. (1973), The pricing of options and corporate liabilities, Journal of Political Economy, 81, 637-654.

[7] Brace A., D. Gatarek, and M. Musiela (1997), The Market Model of Interest Rate Dynamics, Mathematical Finance, 7, 127-55.

[8] Brigo D., and Mercurio F. (2001), Interest Rate Models: Theory and Practice. Springer-Verlag.

[9] Brigo D., Mercurio F., Rapisarda F. (2000), Lognormal-mixture dynamics and calibration to market volatility smiles, working paper, Banca IMI, Milan.

[10] Brigo D., Mercurio F., Rapisarda F. (2004), Smile at the Uncertainty, Risk, May.

[11] Derman E., Kani I. (1994). Riding on a smile, Risk, 7 (2), 32-39.

[12] Delbaen F.; Shirakawa H. (2002), No Arbitrage Condition for Positive Diffusion Price Processes, AsiaPacific Financial Markets, vol. 9, iss. 3-4, pp. 159-168.

[13] Dupire B. (1993), Pricing and hedging with smiles. Reech research paper.

[14] Dupire B.(1994), Pricing with a smile, RISK, Volume 7, No. 1, p. 18-20.

[15] Gatarek, D. (2003), LIBOR Market Model with Stochastic Volatility, Working Paper. Deloitte\&Touche. Downloadable at:

http://papers.ssrn.com/sol3/papers.cfm?abstract_id=359001

[16] Glasserman P., and Kou S.G. (2003): The Term Structure of Simple Forward Rates with Jump Risk; Mathematical Finance, Vol. 13, No. 3, (July 2003), 383-410.

[17] Goldstein R. S. (2000), The Term Structure of Interest Rates as a Random Field, Review of Financial Studies, 13, 365-384.

[18] Heath D., Jarrow R., Morton A. (1992), Bond Pricing and the Term Structure of Interest Rates: A New Methodology. Econometrica 60, 77-105.

[19] Jamshidian, F. (1997), Libor and Swap Market Models and Measures, Finance and Stochastics, 1, 293-330.

[20] Jamshidian F. (1999), Libor Market Model with Semimartingales, NetAnalytics working paper.

[21] Jarrow R., Li H., Zhao F., Interest Rate Caps 'Smile' Too! But Can the LIBOR Market Models Capture it?, Working Paper Cornell University, (2004).

[22] Joshi M. and Rebonato R. (2003), A stochastic-volatility, displaced-diffusion extension of the LIBOR market model. Quantitative Finance 3, 458-469. 
[23] Klöden, P.E. and Platen, E. (1995), Numerical Solutions of Stochastic Differential Equations. Springer, Berlin, Heidelberg, New York.

[24] Miltersen K., K. Sandmann, and D. Sondermann (1997), Closed Form solutions for Term Structure Derivatives with Lognormal Interest Rates, Journal of Finance 409-430.

[25] Piterbarg V. (2003), A Stochastic Volatility Forward LIBOR Model with a Term Structure of Volatility Smiles, Working Paper, Bank of America.

[26] Rebonato R. (2004), Volatility and Correlation, John Wiley \& Sons, 2nd edition.

[27] Rubinstein M. (1991), Pay Now, Choose Later, RISK 4, p. 13.

[28] Santa-Clara P., Sornette D. (2001), The Dynamics of the Forward Interest Rate Curve with Stochastic String Shocks, Review of Financial Studies, 14, 149-185, Spring 2001. 